\title{
Monitoring the abrasive waterjet drilling of Inconel 718 and steel: a comparative study
}

\author{
Huaizhong Li \\ School of Engineering and Built Environment \\ Gold Coast campus, Griffith University, QLD 4222, Australia \\ E-mail: lihuaizhong@gmail.com; h.li@griffith.edu.au \\ Tel.: +61 (7) 555 28252; Fax: +61 (7) 55528065
}

\begin{abstract}
This paper presents an experimental study on abrasive waterjet (AWJ) drilling of Inconel 718 and AISI 1040 steel, with an aim to understand the AWJ hole drilling performance and to investigate the process monitoring strategy by using acoustic emission (AE). In the experiments, pressure levels were set from 200-350 MPa at intervals of $50 \mathrm{MPa}$, and drilling time between 5-30 s at intervals of $5 \mathrm{~s}$. It is found that the hole depth increases along with the drilling time at a slowing down penetration rate. A higher pressure results in a higher penetration rate. While there exists a direct influence of the material property on the drilling efficiency, it has limited significance with regards to the diameter of the hole produced. AE-RMS signal is demonstrated to be able to reflect the decreasing trend of the penetration rate and the influence of the pressure. A strong correlation between the penetration rate and AE-RMS values in AWJ hole drilling is shown through correlation analysis. The AE-RMS is also substantially affected by the material properties. A discrepancy of the AE-RMS under similar condition may suggest an anomaly in this cutting run.
\end{abstract}

Keywords: Abrasive waterjet machining; Inconel 718; hole drilling; monitoring; acoustic emission 


\section{Nomenclature}

$a$

exponential index

$h$

Hole depth

$k$

Empirical coefficient

$t \quad$ Drilling time

AE Acoustic emission

AE-RMS Root mean square of the acoustic emission signal

AWJ Abrasive waterjet

MRR Material removal rate

\section{Introduction}

Inconel 718 has superior mechanical properties like excellent yield strength even at elevated temperature, high oxidation and corrosion resistance, good fatigue life, and has been widely employed in aerospace components, gas turbines, etc. $[1,2]$. However, Inconel 718 is a difficult-to-machine material because of its extreme toughness, high hardness, low thermal conductivity, and high tendency to adhesion and work-hardening [3]. For this material, conventional machining techniques usually show low material removal rate (MRR) and severe cutting tool wear [4], and thus with high machining costs. Fabricating extremely small diameter deep holes often poses significant problems for conventional drilling methods [5].

Abrasive waterjet (AWJ) machining, as an advanced and non-traditional manufacturing technology, has been increasingly used in a wide range of applications, particularly on 
processing difficult-to-cut materials including ceramics, glass, composites, stainless steels, titanium alloys, and superalloys. In AWJ, the mechanical energy of water and abrasive particles is used to cut the material by means of erosion [6]. The waterjet of ultra-high pressure serves as an accelerating medium while the abrasive is responsible for material removal. AWJ is capable of virtually removing any materials through attrition by impacting abrasive particles with high kinetic energy [7]. In comparison with traditional machining technologies, AWJ has various distinct advantages including no thermal distortion, high machining versatility, high flexibility and small cutting forces [8, 9]. It also possesses a capability to drill small holes with high aspect ratios [10]. The tooling cost for abrasive waterjet machining is negligible compared to the cutting tool cost needed in traditional machining [5]. Further, AWJ drilling uses no cutting fluids thus is more environmentally friendly. On the other hand, the cutting ability of the AWJ cutting is limited in terms of the thickness of the target materials that it can penetrate. The harsh working environment and loud noise is a major drawback, and the knowledge of process control to ensure the part quality and dimensional accuracy is a key challenge.

Extensive research has been reported to investigate the machinability and performance of machining different materials by using AWJ, such as stainless steel [11], ceramics $[12,13]$, composites [9], titanium alloys [8, 14], and nickel-based superalloy [15, 16]. There are several major process parameters in AWJ machining that can influence the process performance, including water pressure, nozzle diameter and length, abrasive size and flow rate, the jet feed speed, work material properties, etc. The jet penetration depth in AWJ machining is significantly influenced by these parameters. To produce high quality parts with high productivity, knowledge of the jet penetration depth in terms of the process parameters is very important for process control. There are many research efforts in trying to explore the AWJ machining process and to develop predictive models that can quantify the influence of 
the process parameters on the jet footprint, such as [17, 18]. Zeng and Kim [19] developed a comprehensive model for AWJ transverse cutting parameter prediction, which makes the cutting speed prediction more realistic. It has become a very universal model for AWIJ cutting process of many cutting system. Ramulu et al. [20] reviewed the existing models for AWJ drilling of blind holes. It was reported that most of the models discussed were based in one form or another on the conservation of the energy of the jet and its balance by the material removal rate. Most of the models have not taken into account the backflow of the jet in the AWJ hole drilling process. Rabani et al. [7] proposed an Iterative Learning Control based approach to predict the jet penetration depth in AWJ milling. It was pointed out that the jet penetration is difficult to control through conventional feedback control techniques due to lack of precise measurements of process outputs and monitoring systems. Due to the process complexity and variety of work materials, monitoring the AWJ machining process and the jet penetration depth has become increasingly important as a possible process supervision solution.

Some attempts of using acoustic emission (AE) sensors to monitor the AWJ machining process have been reported [21, 22]. Acoustic emission is a transient elastic stress wave emitted and propagated inside a material, due to the sudden release of stored strain energy in the material, such as the occurrence of fracture, plastic deformation, crack formation, and phase transform. Choi and Choi [23] investigated AE signals generated from AWJ machining of alumina ceramics in view of establishing an experimental relationship with the parameters of material removal process. Sutowski et al. [24] proposed the existence of relationships between the emitted AE signal and the quality of the cut surfaces from an analysis of experimental results during AWJ cutting of aluminium alloy 5251. Mokhtar et al. [25] analysed $\mathrm{AE}$ produced during abrasive waterjet machining of aluminium 6061 and stainless steel 304 and reported that the AE spectrums correlated with different feed rates and surface 
qualities. Pahuja and Ramulu [26] employed AE and vibrational energy to monitor abrasive waterjet process. They calculated the time domain root mean square value of the and developed an empirical model to correlate acoustic energy with jet energy and cross-sectional area of the AWJ milled slot. Hassan et al. [27] found that the root mean square of the AE energy (AE-RMS) increases linearly with an increase in the depth of cut when cutting AISI $1018 \mathrm{CD}$ carbon steel using AWJ, and suggested that AE-RMS could be used for on-line monitoring of the depth of cut in AWJ cutting. Rabani et al. [22] proposed an energy based monitoring concept for AWJ milling of titanium alloy Ti6Al4V to supervise the jet penetration depth using AE energy transfer rate.

However, there is a lack of comprehensive study to investigate the influence of the process parameters on the penetration depth in AWJ cutting of Inconel 718, and the correlation of AE signals in the process. As small hole drilling on Inconel 718 is an important and challenging task for manufacturing industry, there is a need to study the AWJ drilling process and associated process monitoring. A deeper understanding of how acoustic emission is affected by the depth of penetration on Inconel 718 will allow a possible on-line monitoring and controlling of the penetration process, and allow significant improvements to be made in the performance of abrasive waterjet machining. The objective of this paper is to understand the AWJ hole drilling performance and to investigate the process monitoring by using AE signals, which is conducted through an experimental study on AWJ drilling of Inconel 718, and also on AISI 1040 steel as a comparison. The reason of using AISI 1040 as a benchmarking material is that AISI 1040 is a commonly used medium strength steel in metal machining workshops. It is with medium tensile strength and suitable for making shafts, couplings, studs, keys etc. The machinability rating of AISI 1040 is 60, while it is 12 for Inconel 718. 


\section{Experimentation}

Experimental work on abrasive waterjet drilling of Inconel 718 and steel was conducted by using an abrasive waterjet cutting system with five major modules, i.e., a Flow 5X-60 intensifier pump to provide high-pressure water, a Paser 3 abrasive delivery system which supplies abrasives through the delivery line to the focusing tube (or the nozzle), a cutting head in which the high pressure water mixes with the abrasive media to create a collimated jet through a nozzle that performs the machining process, a 6-axis ABB IRB2400 robot manipulator to hold the cutting head nozzle in place and can be controlled to follow a designed tool path to permit flexible machining operations, and a water catchment tank to contain the waterjet and any debris from the machining process, as shown in Fig. 1 (a).

(a)

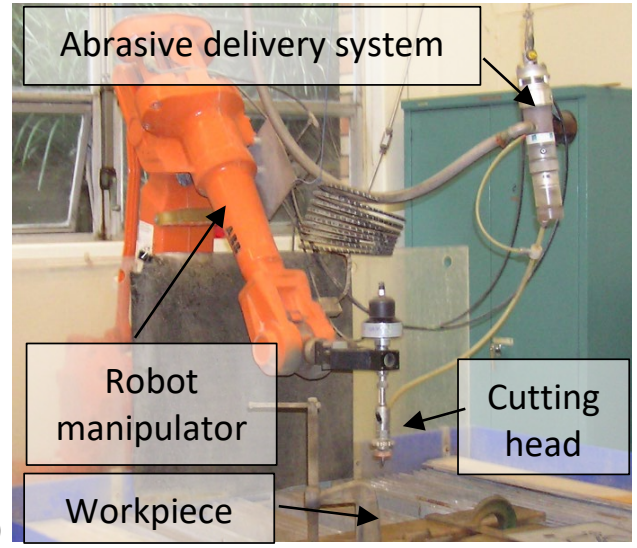

(b)

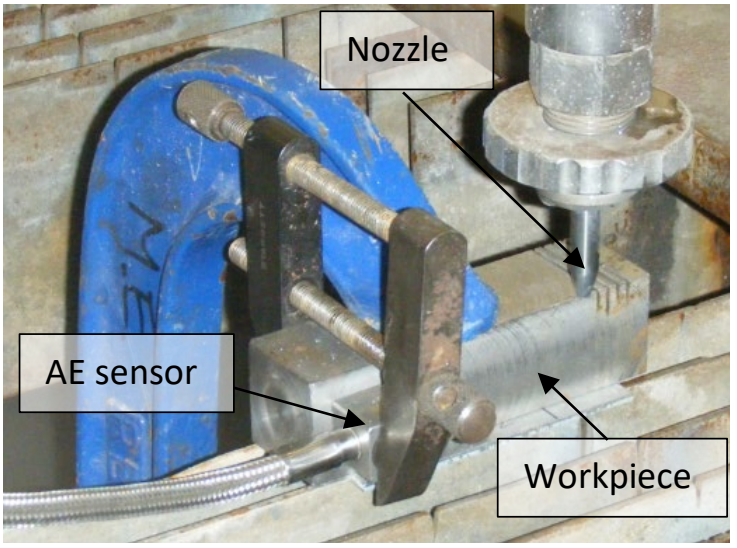

(c)

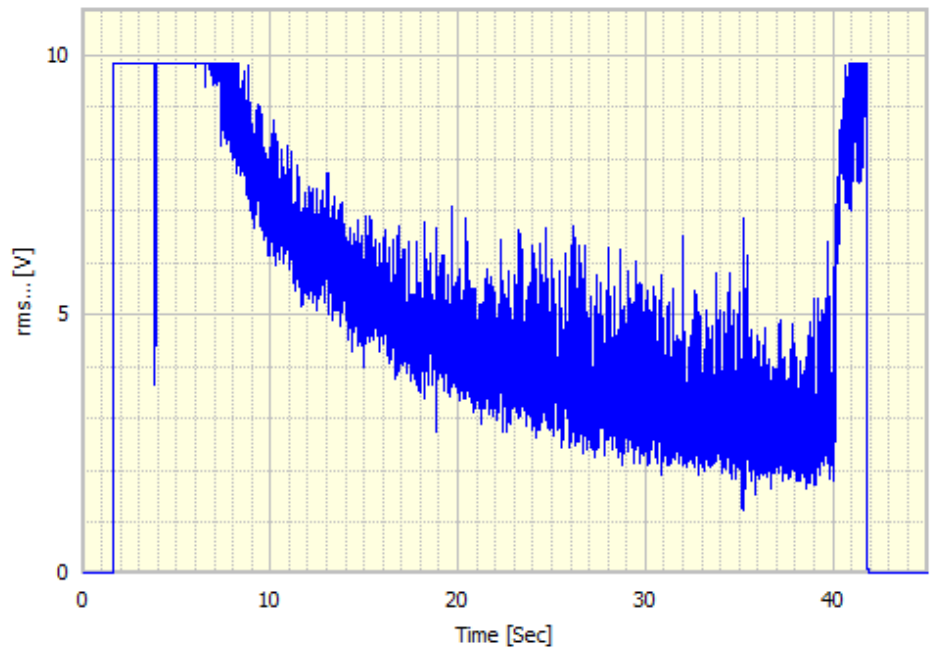


(d)

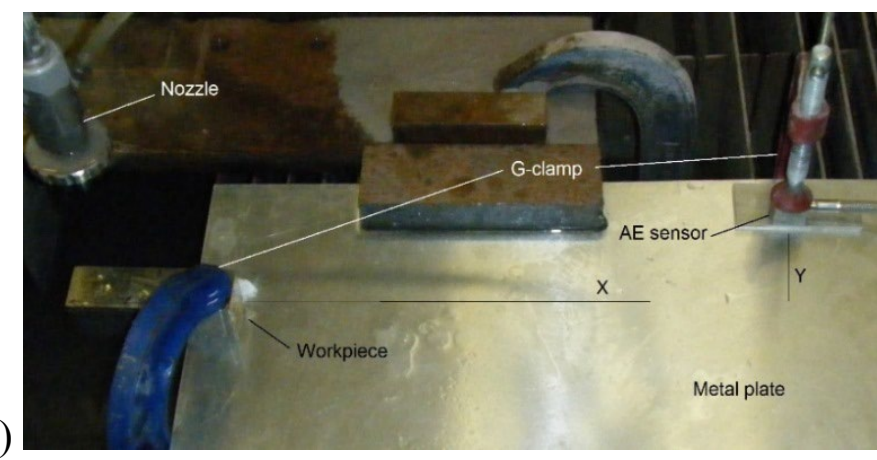

Fig. 1. (a) The robot manipulator and the cutting head nozzle for the AWJ system; (b) Locations of workpiece and the AE sensor in the preliminary testing; (c) Acquired AE signal with sensor saturation;

(d) Final locations of workpiece and the $\mathrm{AE}$ sensor

Table 1. Inconel 718 chemical composition (\%)

\begin{tabular}{cccccccccc}
\hline $\mathrm{C}$ & $\mathrm{Cr}$ & $\mathrm{Ni}$ & $\mathrm{Mo}$ & $\mathrm{Al}$ & $\mathrm{B}$ & $\mathrm{Cu}$ & $\mathrm{Mn}$ & $\mathrm{Si}$ & $\mathrm{Nb}$ \\
\hline 0.04 & 19.0 & 52.5 & 3.0 & 0.90 & 0.006 & 0.1 & 0.35 & 0.35 & 5.1 \\
\hline
\end{tabular}

Table 2. AISI 1040 Carbon Steel chemical composition (\%)

\begin{tabular}{ccccc}
\hline $\mathrm{Fe}$ & $\mathrm{Mn}$ & $\mathrm{C}$ & $\mathrm{S}$ & $\mathrm{P}$ \\
\hline $98.6-99$ & $0.60-0.90$ & $0.370-0.440$ & $\leq 0.050$ & $\leq 0.040$ \\
\hline
\end{tabular}

The materials used in the testing were Inconel 718 and AISI 1040 mild carbon steel. The Inconel 718 was in hardened and tempered square bar. According to a datasheet provided by the supplier, the chemical composition of the Inconel 718 is listed in Table 1, and the mechanical properties in the room temperature include a tensile strength of $965 \mathrm{MPa}$, yield point of $550 \mathrm{MPa}$, and an elongation of $30 \%$. The nominal Brinell hardness is $331 \mathrm{HB}$. The workpieces were prepared into rectangular blocks in sizes of $100 \mathrm{~mm} \times 30 \mathrm{~mm} \times 30 \mathrm{~mm}$. For comparison, unalloyed medium carbon steel (AISI 1040) workpiece with the same size was also prepared. For AISI 1040 steel, the tensile strength is $620 \mathrm{MPa}$, the yield strength is 415 $\mathrm{MPa}$, and the Brinell hardness is $201 \mathrm{HB}$ [28]. The chemical composition of AISI 1040 steel is listed in Table 2. 
A Kistler type 8152B111 acoustic emission sensor was used in the experiments to monitor the AWJ cutting process. The AE sensor was connected with a type 5125 AE-Piezotron coupler which was functioned to provide a continuous supply of power to the sensor and process the signal from the sensor. The coupler provided outputs of the raw AE signals and also the processed AE RMS signals, which was recorded by a Prosig P8004 data acquisition system. The gain jumper of $\mathrm{x} 1$ was used in the coupler. The coupler had a bandpass filter obtained by the series connection of one high-pass filter $50 \mathrm{kHz}$ and one low-pass filter 1000 $\mathrm{kHz}$. A built-in RMS converter with an integration time constant of $1.2 \mathrm{~ms}$ was employed in the coupler to output the AE RMS signal.

Preliminary drilling testing was conducted with the AE sensor attached directly to the workpiece as shown in Fig. 1(b), in which the AE sensor had direct contact with the workpiece. In such a location, the measured AE signal was too high and sensor saturation was encountered. The acquired AE-RMS signal under a trial drilling test with $200 \mathrm{MPa}$ pressure is shown in Fig. 1(c). There exists a horizontal straight line at $10 \mathrm{~V}$ from $2.5 \mathrm{~s}$ to above $10 \mathrm{~s}$, indicating the saturation status of the AE sensor. It is clear that the AE-RMS signal exceeded the maximum output voltage value of the sensor $(10 \mathrm{~V})$ and got saturated due to the high acoustic emission intensity in the AWJ drilling. To remedy the saturation problem, we had to locate the AE sensor away from the workpiece by using a steel plate to mount both the sensor and the workpiece through G-clamps, as shown in Fig. 1(d). For all the testing presented in this paper, the distance of the sensor from the workpiece edge was fixed as $\mathrm{X}=360 \mathrm{~mm}$ and $\mathrm{Y}=45 \mathrm{~mm}$ (see Fig. 1(d)). The added surface contact between the workpiece / metal plate / AE sensor, and the increased AE signal transmission path as well, contributed to a certain level of signal attenuation, thus the sensor saturation was avoided. 
(a)

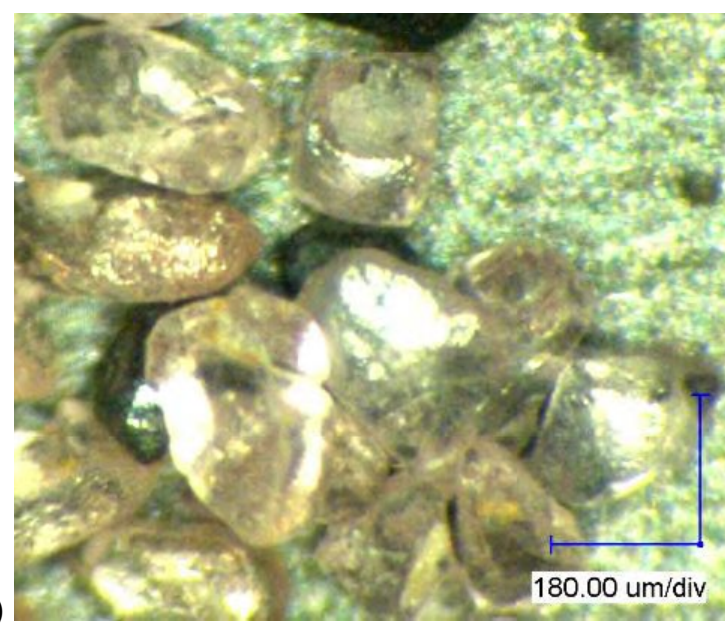

(b)
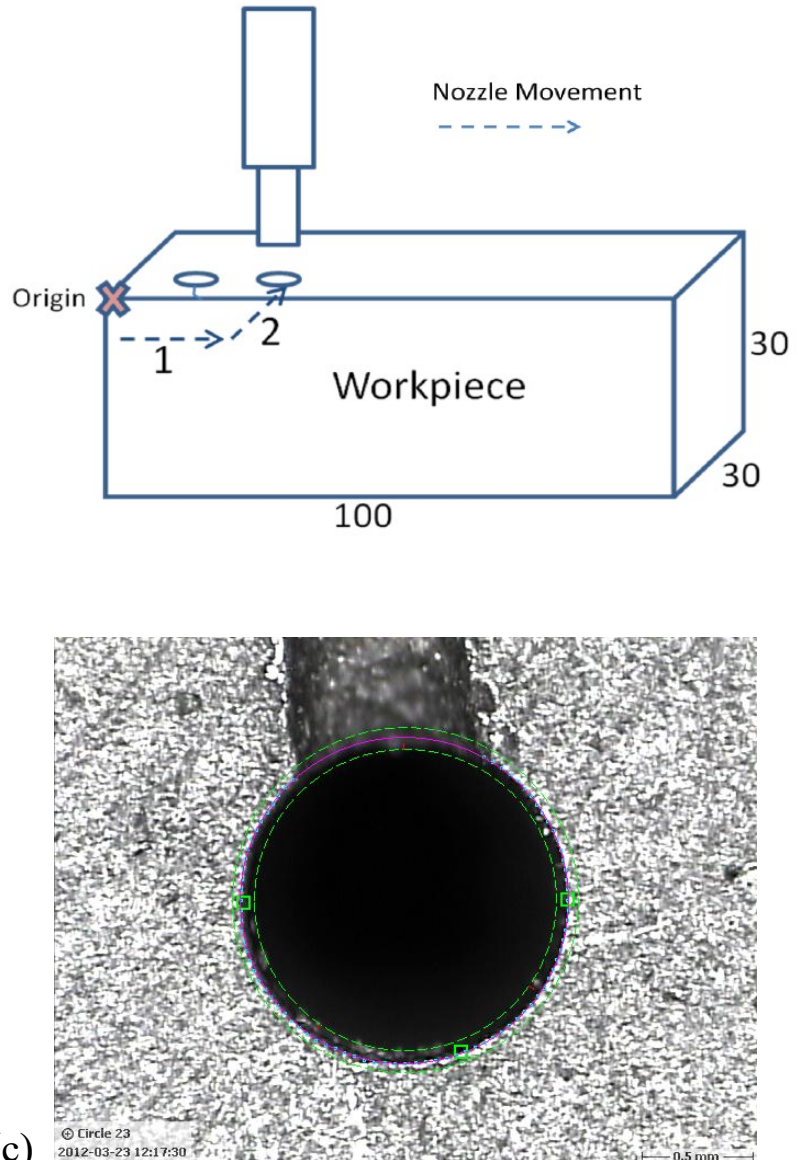


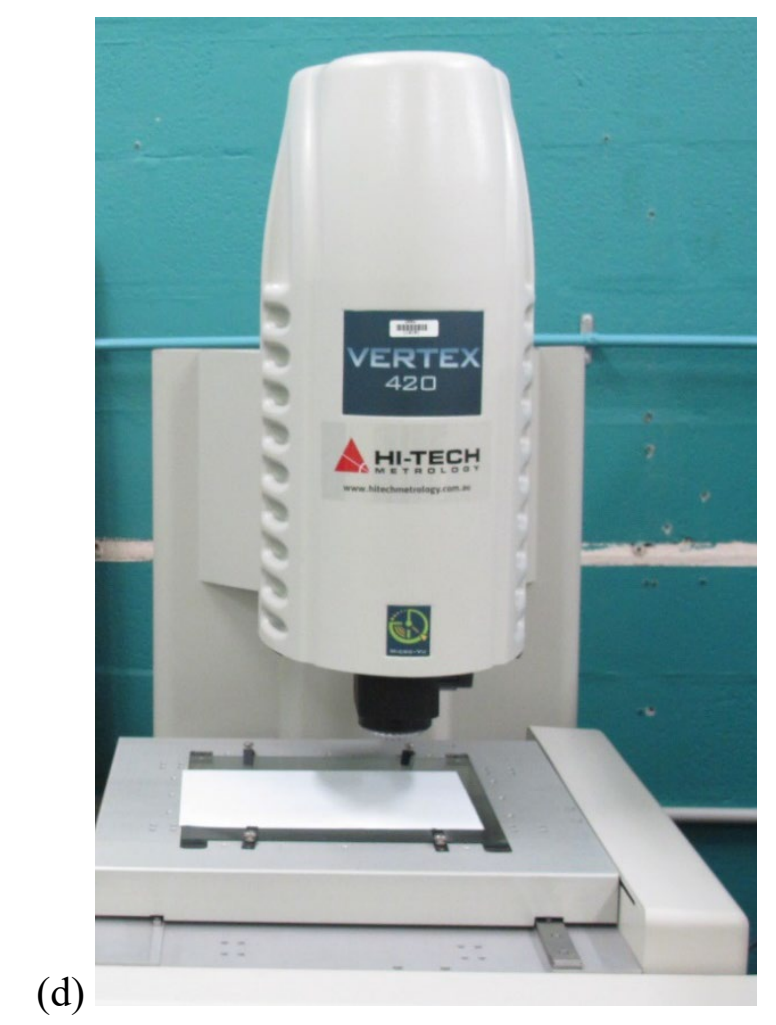

Fig. 2. (a) Sample photo of the garnet particles under microscope; (b) Nozzle movement procedures in the AWJ drilling experiments; (c) a resultant blind hole; (d) Vertex 420 for hole diameter measurement

A large number of process variables can affect the AWJ machining process. The abrasives used throughout the experiments were GMA garnet sand with a mesh number of \#80. The average abrasive size is $180 \mu \mathrm{m}$. A sample photo of the garnet particles under microscope is shown in Fig. 2(a) [29]. It can be seen that most of particles are in irregular shapes far away from spherical shape but without sharp edges. The abrasive mass flow rate can influence the material removal rate in AWJ machining and in general, supplying more abrasives should remove more material. However, increasing abrasive mass flow rate can be countered by a decrease in abrasive particle velocity when the water pressure is fixed due to the momentum transfer principle. Further, more abrasive particles may collide with each other and cause a waste of kinetic energy and scatter of particle motion direction. There should be an optimum abrasive mass flow rate to achieve the maximum material removal rate. In a recent study to cut AISI4340 steel, Li [29] reported that the optimum abrasive mass flow rate was around 
420 g.min ${ }^{-1}$ (or 7 g.s s $^{-1}$ ) when jet pressure was $380 \mathrm{MPa}$, and below 228 g.min ${ }^{-1}\left(3.8\right.$ g.s ${ }^{-1}$ ) while at $200 \mathrm{MPa}$. In this study, an abrasive mass flow rate of 333 g.min ${ }^{-1}$ (or 5.55 g.s ${ }^{-1}$ ), which is close to the middle of 228 and 420 g. $\mathrm{min}^{-1}$. The abrasives mass flow rate of the Abrasive Delivery System was controlled by using orifice discs of different diameters. The abrasive mass flow rate of 333 g. $\mathrm{min}^{-1}$ was maintained by using only one orifice disc corresponding to the specified flow rate value.

The pressure and drilling time were chosen as the main variables to control in the AWJ drilling process, each of which was selected at multiple levels. Preliminary experiments were performed to determine the range of the operating parameters. It was determined that pressure levels at intervals of $50 \mathrm{MPa}$ from 200-350 MPa and drilling time between 5-30 s at intervals of $5 \mathrm{~s}$ for AWJ drilling were used. Specifically, the pressure levels included 200, 250, 300, and $350 \mathrm{MPa}$, while the drilling times included 5, 10, 15, 20, 25, and $30 \mathrm{sec}$. Therefore, a total number of 24 drilling testings were conducted on each material. Experiments were performed sequentially following the experiment numbers. Drilling was conducted on both Inconel 718 and 1040 steel to study the effect of material properties on the AWJ drilling. Other fixed parameters are displayed in Table 3 below. In the AWJ drilling experiments, as illustrated in Fig. 2 (a), the nozzle head was made to traverse along "1", before the jet was switched on and approach the pre-determined location of the hole. Drilling was performed at the preset pressure level and drilling time before the jet was switched off and returned to the origin point. The photo of a resultant hole is shown in Fig. 2 (b). The depth of the drilled blind holes was measured by using a Mitutoyo digital height gauge calliper with an attached steel needle. Measurement of the hole diameter was taken using a Vertex 420 non-contact vision measuring system from MicroVu, as shown in Fig. 2 (d). 
Table 3. Fixed Process Parameters in AWJ drilling

\begin{tabular}{ll}
\hline Ruby Orifice Diameter & $0.254 \mathrm{~mm}$ \\
\hline Focusing Tube Diameter & $0.762 \mathrm{~mm}$ \\
\hline Focusing Tube Length & $76.2 \mathrm{~mm}$ \\
\hline Abrasive Material & Garnet \\
\hline Abrasive Size & 80 mesh $(180 \mu \mathrm{m}$ on average $)$ \\
\hline Abrasive mass flow rate & 333 g.min ${ }^{-1}$ \\
\hline Stand-off Distance & $3 \mathrm{~mm}$ \\
\hline
\end{tabular}

\section{Results and discussions}

\subsection{AWJ hole drilling performance}

\subsubsection{Hole depth}

The evolution of hole depth vs. the drilling time for the two work materials under different pressures is shown in Fig. 3 (a) and (b) respectively. It is clear that the hole depth increases along with the drilling time. The higher the water pressure, the deeper the hole depth. AWJ is well accepted to be able to machine almost any engineering material, irrespective of material properties [30]. As both materials are ductile in nature, the material removal mechanism is similar. Although somebody considered that AWJ is going to perform almost identically in almost any material [31], however, it has been reported that different materials are found to possess different removal rates in machining by using AWJ [32]. This is confirmed in the current study. For ductile-behaving materials, the material erosion mechanism due to microparticle impacting is usually attributed to micro-cutting. It may include a cutting wear mode occurring at low-impact angles, and a deformation wear mode at high-impact angles. When an abrasive particle impacts on a workpiece, it acts on the target surface with two force 
components, i.e., normal to and perpendicular to the surface respectively. The normal force component facilitates an indentation in the workpiece and causes deformation wear, while the tangential component promotes shearing stresses which may create microchips from the workpiece or cause a ploughing action to the surface [33]. Low-cycle fatigue was also considered as a possible mechanism of solid-particle erosion for normal impact [34]. In the AWJ drilling process, the abrasive particles impact on the hole-bottom nearly perpendicular to it. Hence the erosion mode on the bottom is mostly deformation wear. When the particles are bounced back from the bottom, they may act on the hole-wall at low-impact angles, then cutting wear mode happens on the wall and enlarges the hole-diameter. As such, the material removal rate is associated with the material properties such as a plastic response character of the material determined by its flow stress, and the toughness as well. Due to the difference in terms of the strength, the hole depth on the steel as shown in Fig. 3 (b) is much higher than that on the Inconel under the same condition.

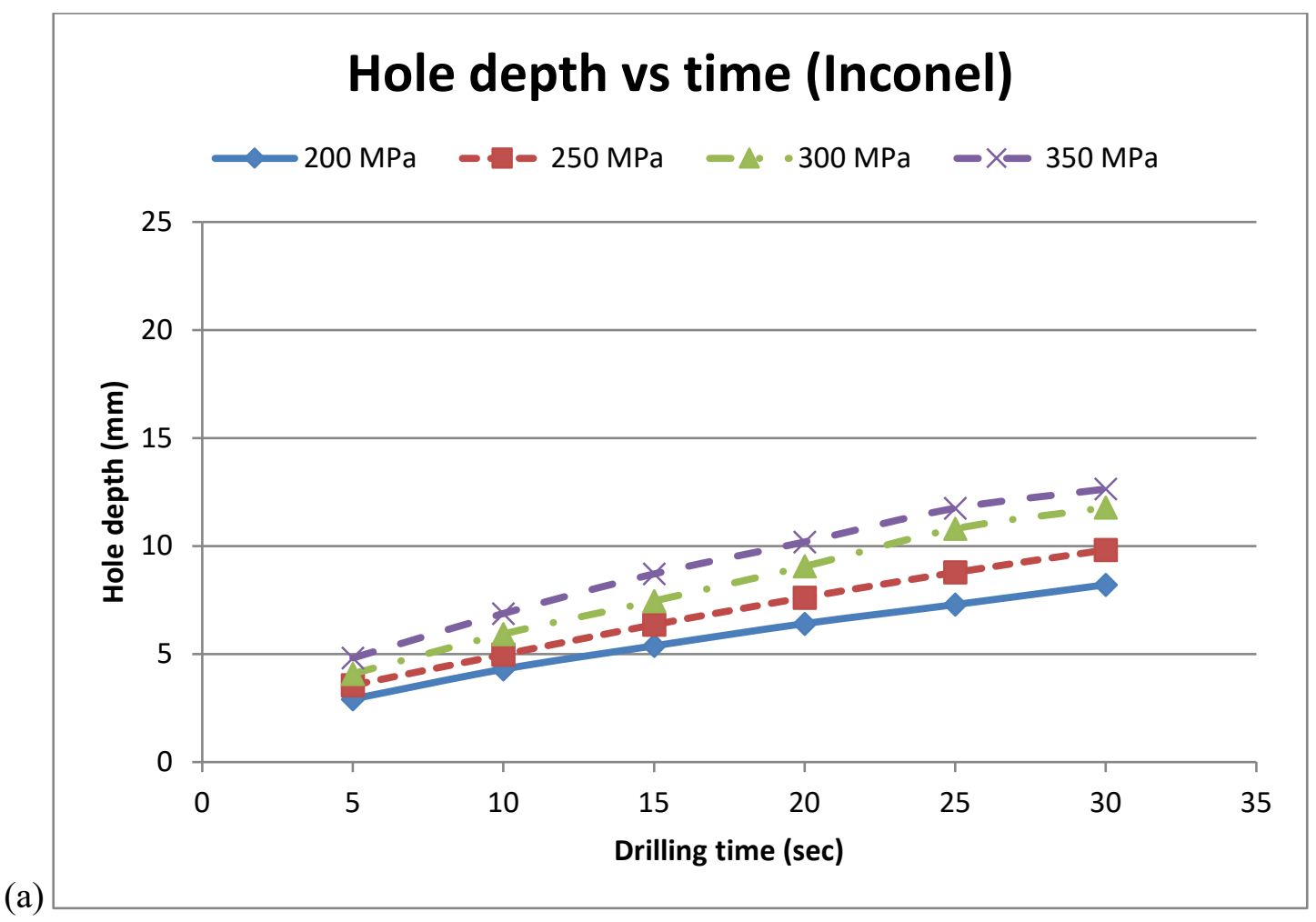




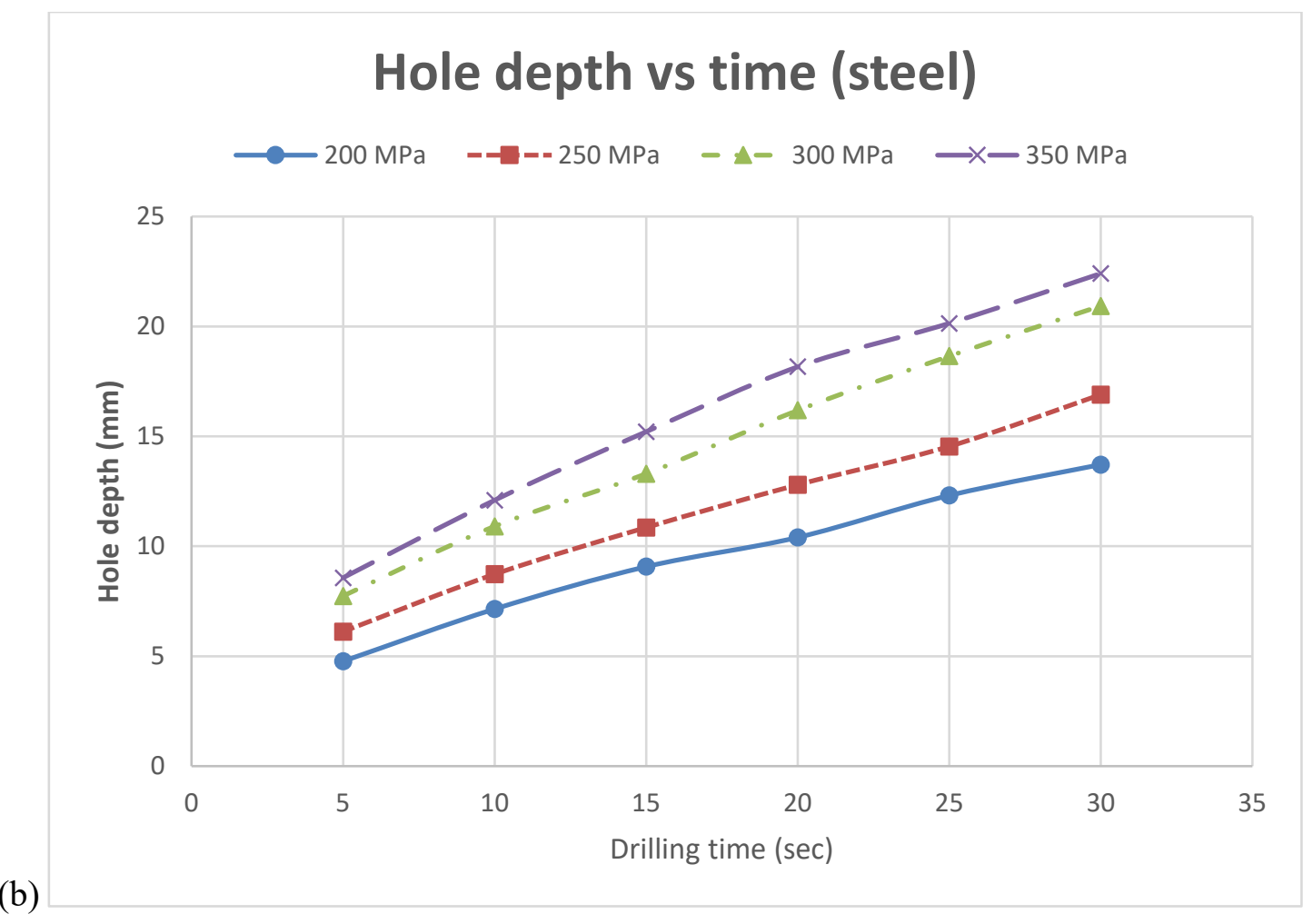

Fig. 3. Hole depth vs. the drilling time for (a) Inconel 718, and (b) 1040 steel, under different conditions

Table 4. Ratio of the hole depth for the two materials (steel / Inconel) under each condition

\begin{tabular}{cccccr}
\hline \multirow{2}{*}{ Time (s) } & \multicolumn{5}{c}{ Pressure (MPa) } \\
\cline { 2 - 5 } & 200 & 250 & 300 & 350 & \\
& 1.63 & 1.72 & 1.89 & 1.78 & 1.76 \\
5 & 1.66 & 1.75 & 1.84 & 1.76 & 1.75 \\
10 & 1.69 & 1.71 & 1.78 & 1.75 & 1.73 \\
15 & 1.62 & 1.68 & 1.79 & 1.78 & 1.72 \\
20 & 1.69 & 1.66 & 1.73 & 1.71 & 1.70 \\
25 & 1.67 & 1.72 & 1.78 & 1.77 & 1.74 \\
30 & 1.66 & 1.71 & 1.80 & 1.76 & 1.73 \\
\hline Average
\end{tabular}

Table 5. Ratio of material properties (Inconel/ steel)

\begin{tabular}{lccc}
\hline Material & $\begin{array}{c}\text { Tensile strength } \\
(\mathrm{MPa})\end{array}$ & $\begin{array}{c}\text { Yield Strength } \\
(\mathrm{MPa})\end{array}$ & $\begin{array}{c}\text { Hardness } \\
(\mathrm{HB})\end{array}$ \\
\hline Inconel 718 & 965 & 550 & 331 \\
\hline 1040 steel & 620 & 415 & 201 \\
\hline Ratio (Inconel/steel) & 1.56 & 1.33 & 1.65 \\
\hline
\end{tabular}


The ratio of the hole depth for the two materials (steel / Inconel) under each condition is listed in Table 4. The ratio is in a range of 1.62-1.89. It can be observed that the variation of the ratios is very limited. Under each water pressure, a change of drilling time does not cause an obvious change of the depth ration. However, different water pressure can give rise to a slight variation of the depth ratio. This phenomenon might be attributed to the variation of erosion rate of the two materials corresponding to different particle energy levels. Table 5 shows the ratios of some material properties for the two materials (Inconel/ steel), including tensile strength, yield Strength, and Brinell hardness. It is interesting to note that the hole depth ratio of the two materials is very close to the inverse of their hardness ratio. AWJ drilling of a softer material (1040 steel in this case) resulted in a deeper hole. It provides a support to the opinion that material hardness influences the material erosion significantly. Further, it is seen that higher material strength resulted in lower erosion rate. For the two materials used in this study, the elongation is very close $(25 \%-30 \%)$. Since increasing hardness can cause a reduction of the ductility which may contribute to a higher erosion rate consequently, consideration of material toughness as an erosion rate indicator might be a good idea and worth further investigation to establish a correlation for toughness and its erosion performance.

It is also observed that the relationship between the drilling depth and drilling time is nonlinear. The increasing rate of the hole depth was slightly slowing down. Several regression models were assessed to represent the relationship between the hole depth and the drilling time, including the linear model, power model, exponential model, and logarithmic model. It is found that the power regression curve exhibits the best correlation coefficient for the experimental data, in which the hole depth $h$ can be approximated as

$h=k t^{a}$ 
where the hole depth $h$ is in $\mathrm{mm}, t$ is the drilling time in s, and $k$ and $a$ represent the empirical coefficient and exponential index that are defined by the power regression curve. The coefficient of the power regression curve, $k$, is highly dependent on the material properties.

\subsubsection{Penetration rate}

Due to the lack of a direct mean to measure the hole depth in real time during the AWJ drilling process, in this study, the drilling time under each pressure level was set as 5, 10, 15, 20,25 , and $30 \mathrm{~s}$. A segmented estimation of the penetration rate for each period of 5 seconds can be made based on the measured hole depth accordingly. The result is shown in Fig. 4 (a) and (b) for drilling Inconel 718 and the steel respectively. It is seen that under a higher water pressure, the penetration rate is also higher. In AWJ machining, the material removal is mainly caused by the impact of solid abrasive particles with high velocity. The process of separating the ductile materials such as the Inconel and steel used in this study is considered to be caused by micro-cutting and separating by material plastic deformation. When the abrasive jet impinges on the surface of the workpiece material in an AWJ drilling operation, due to the high impact, some volume of the material is removed from the target workpiece which consumes the kinetic energy of the jet. It is estimated that the velocity of a waterjet is proportional to the square root of water pressure [35]. Under a higher pressure level, the jet velocity is also higher, as such, a higher kinetic energy level of the jet is obtained and causes a higher penetration rate and thus a higher material removal rate. 
Penetration rate in drilling Inconel 718

$\rightarrow 200 \mathrm{MPa} \rightarrow-250 \mathrm{MPa} \rightarrow-300 \mathrm{MPa} \rightarrow-350 \mathrm{MPa}$

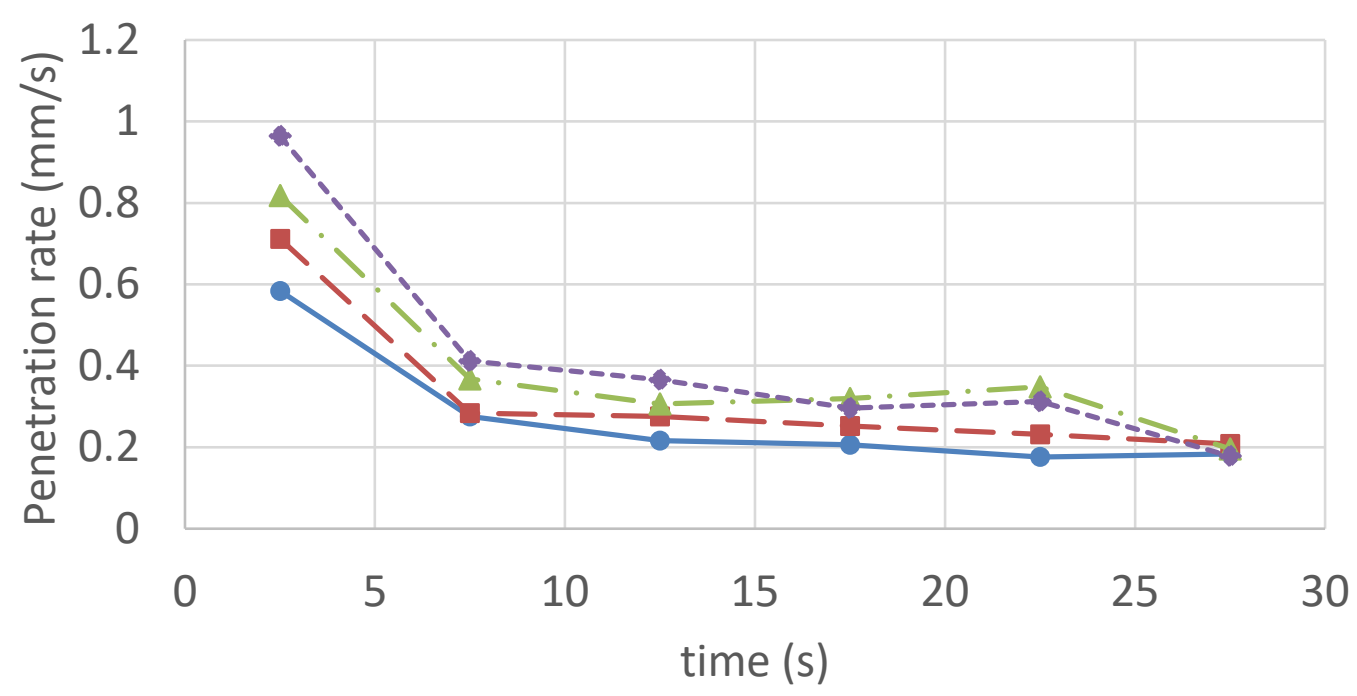

(a)

\section{Penetration rate in drilling steel}

$\longrightarrow 200 \mathrm{MPa} \rightarrow-250 \mathrm{MPa} \rightarrow \cdot-300 \mathrm{MPa} \rightarrow-350 \mathrm{MPa}$

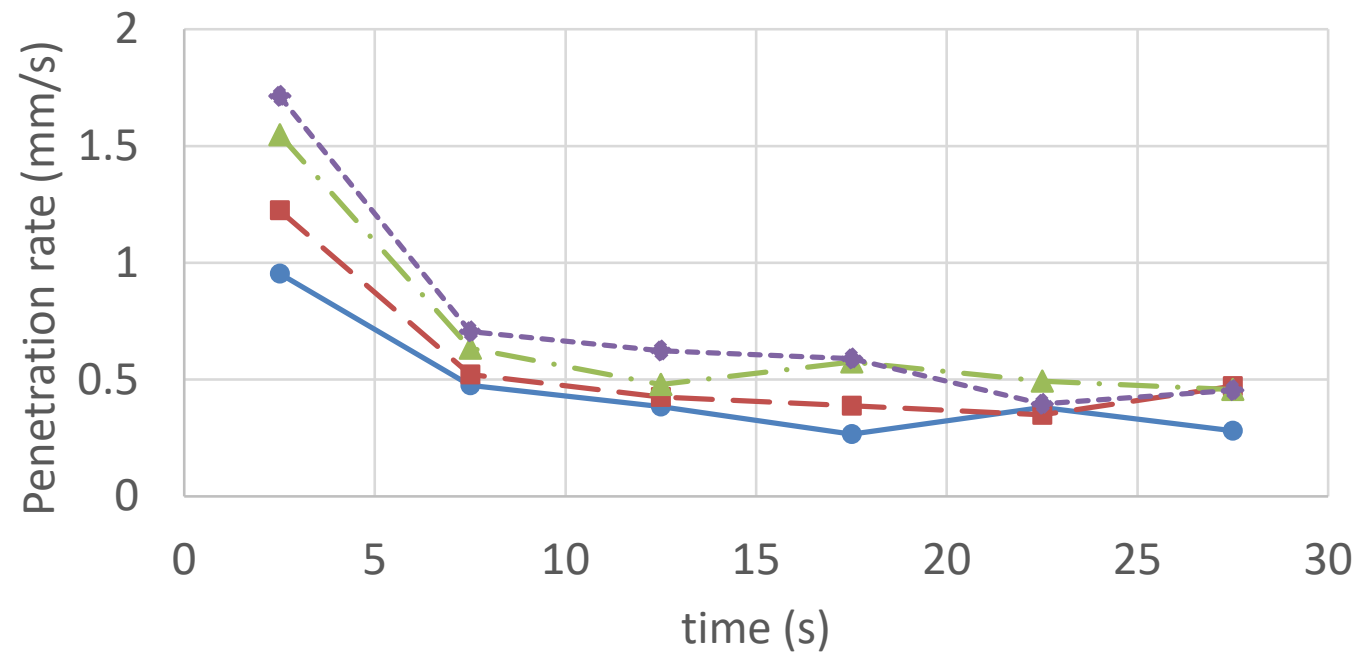

(b)

Fig. 4. Segmented estimation of the penetration rate for AWJ drilling of (a) Inconel 718, and (b) 1040 steel 
It can be seen that the penetration rate decreases along with the drilling time and the increasing hole depth. This phenomenon can be attributed to the fact that to drill into a deeper hole, more kinetic energy of the jet is wasted due to the necessity to travel through a longer distance in the blind hole and to overcome the resistance caused by the backflow. With an increasing depth, the velocity of the abrasive particles in the jet decreases due to the existence of viscous drag force and a backflow of the impacting jet, and therefore results in a lower material removal rate.

On the other hand, the penetration rate is also significantly related to the work material property. It has been reported that the erosion rate of the target metal correlates positively with grain size and negatively with hardness but this correlation is nonlinear [15]. The penetration rate for the steel is much higher than that for the Inconel under the same pressure, which can be attributed to the difference in the strengths of the two materials. The tougher the work material, the more difficult for the erosion to happen.

It should be pointed out that in this study, only one type of abrasive material, GMA garnet sand with a mesh number of \#80, was used in the experiments. Although it has been reported in a survey that $90 \%$ of the AWJ machining was done by using garnet instead of artificial abrasives, various types of abrasives do effect the AWJ machining performance significantly [36]. Khan and Haque [37] studied the performance of garnet, aluminum oxide and silicon oxide during AWJ machining of glass. It was found that the garnet abrasives produce the smallest width of cut and larger taper of cut, perhaps due to its low hardness among these three abrasives. The higher the hardness of an abrasive, the higher its cutting ability. Fowler et al. [38] investigated AWJ milling of Ti6A14V with different abrasives, including white and brown aluminium oxide, garnet, glass beads and steel shot. It was demonstrated that the ratio between the hardness of the workpiece and the abrasive is more important than particle shape. Material removal rate and surface roughness increased when particle hardness is increased. 
Axinte et al. [39] reported that the material removal rates vary significantly with the employment of different types of abrasives. The jet penetration capability increased with the hardness of the abrasives. It would be interesting to further investigate the machining behavior of AWJ drilling of Inconel 718 using some harder abrasives, such as aluminium oxide $\left(\mathrm{Al}_{2} \mathrm{O}_{3}\right)$, silicon carbide ( $\left.\mathrm{SiC}\right)$ and diamond. However, the usage of harder abrasives might result in a rapid wear in the inside of focusing nozzle and the increased cost of the abrasives. Therefore, it requires further work to address these issues.

\subsubsection{Hole diameter}

Table 6. Measured diameter and circularity under different cutting conditions for Inconel 718

\begin{tabular}{|c|c|c|c|c|c|c|c|c|c|}
\hline \multirow{2}{*}{$\begin{array}{c}\text { Pressure } \\
\text { (MPa) }\end{array}$} & \multirow{2}{*}{$\begin{array}{l}\text { Time } \\
(\mathrm{s})\end{array}$} & \multicolumn{4}{|c|}{ Diameter (mm) } & \multicolumn{4}{|c|}{ Circularity (mm) } \\
\hline & & $\begin{array}{c}\text { Reading } \\
1 \\
\end{array}$ & $\begin{array}{c}\text { Reading } \\
2\end{array}$ & $\begin{array}{c}\text { Reading } \\
3\end{array}$ & Average & $\begin{array}{c}\text { Reading } \\
1 \\
\end{array}$ & $\begin{array}{c}\text { Reading } \\
2\end{array}$ & $\begin{array}{c}\text { Reading } \\
3\end{array}$ & Average \\
\hline \multirow{6}{*}{200} & 5 & 1.311 & 1.318 & 1.317 & 1.315 & 0.021 & 0.242 & 0.019 & 0.094 \\
\hline & 10 & 1.410 & 1.406 & 1.408 & 1.408 & 0.021 & 0.026 & 0.022 & 0.023 \\
\hline & 15 & 1.467 & 1.467 & 1.467 & 1.467 & 0.024 & 0.025 & 0.018 & 0.022 \\
\hline & 20 & 1.525 & 1.537 & 1.524 & 1.529 & 0.022 & 0.025 & 0.020 & 0.022 \\
\hline & 25 & 1.563 & 1.561 & 1.562 & 1.562 & 0.025 & 0.022 & 0.023 & 0.023 \\
\hline & 30 & 1.600 & 1.597 & 1.596 & 1.598 & 0.026 & 0.025 & 0.026 & 0.026 \\
\hline \multirow{6}{*}{250} & 5 & 1.306 & 1.339 & 1.335 & 1.327 & 0.025 & 0.239 & 0.023 & 0.096 \\
\hline & 10 & 1.423 & 1.425 & 1.438 & 1.428 & 0.029 & 0.023 & 0.026 & 0.026 \\
\hline & 15 & 1.503 & 1.503 & 1.508 & 1.505 & 0.030 & 0.025 & 0.024 & 0.026 \\
\hline & 20 & 1.567 & 1.566 & 1.569 & 1.567 & 0.021 & 0.018 & 0.021 & 0.020 \\
\hline & 25 & 1.614 & 1.609 & 1.614 & 1.612 & 0.018 & 0.017 & 0.019 & 0.018 \\
\hline & 30 & 1.665 & 1.640 & 1.646 & 1.650 & 0.021 & 0.021 & 0.027 & 0.023 \\
\hline \multirow{6}{*}{300} & 5 & 1.345 & 1.344 & 1.348 & 1.345 & 0.019 & 0.020 & 0.026 & 0.022 \\
\hline & 10 & 1.446 & 1.451 & 1.445 & 1.447 & 0.024 & 0.025 & 0.025 & 0.025 \\
\hline & 15 & 1.520 & 1.516 & 1.519 & 1.518 & 0.020 & 0.018 & 0.019 & 0.019 \\
\hline & 20 & 1.586 & 1.584 & 1.569 & 1.579 & 0.034 & 0.023 & 0.028 & 0.028 \\
\hline & 25 & 1.623 & 1.623 & 1.623 & 1.623 & 0.020 & 0.026 & 0.025 & 0.024 \\
\hline & 30 & 1.670 & 1.669 & 1.666 & 1.668 & 0.039 & 0.023 & 0.027 & 0.030 \\
\hline \multirow{6}{*}{350} & 5 & 1.374 & 1.373 & 1.372 & 1.373 & 0.017 & 0.020 & 0.016 & 0.018 \\
\hline & 10 & 1.474 & 1.472 & 1.473 & 1.473 & 0.015 & 0.024 & 0.016 & 0.018 \\
\hline & 15 & 1.545 & 1.543 & 1.547 & 1.545 & 0.020 & 0.014 & 0.019 & 0.018 \\
\hline & 20 & 1.613 & 1.612 & 1.616 & 1.614 & 0.023 & 0.021 & 0.056 & 0.033 \\
\hline & 25 & 1.657 & 1.658 & 1.661 & 1.659 & 0.017 & 0.025 & 0.025 & 0.023 \\
\hline & 30 & 1.701 & 1.712 & 1.713 & 1.709 & 0.151 & 0.022 & 0.029 & 0.067 \\
\hline
\end{tabular}


The variation of the hole diameter vs drilling time for both materials is shown in Fig. 5. Although the internal diameter of the nozzle is only $0.762 \mathrm{~mm}$, the resultant hole diameters are much larger than it, roughly two times in size. Even with the jet flow expansion considered [40], in AWJ drilling, the hole generated is significantly wider than the jet stream. This phenomenon is due mostly to the additional wall erosion resulting from the backflow, which is the forceful upward ejection of the jet out of the blind cavity. A list of the measured average hole diameter and circularity under different cutting conditions for Inconel 718 is provided in Table 6. It can be seen that an average ratio of the circularity over the hole diameter is about $2 \%$, which demonstrated relatively good roundness of the machined holes.

With the cutting time increased from $5 \mathrm{~s}$ to $30 \mathrm{~s}$, there is a further increment of the hole diameter by about $20-30 \%$. The phenomenon can be explained by two reasons. Firstly, while the majority of abrasive particles impact the target material at the hole bottom by an almost perpendicular angle, rebound flow is generated. The back flow of the abrasive jet squeezes out from the blind hole which causes further erosion of the hole wall at low-impact angles and thus enlarges the hole diameter. This should be the major reason. Secondly, the waterjet impact is a highly dynamic process. There exists a certain level of flow fluctuation and slight variations of the jet expansion angle. Such variation of jet expansion causes a change of the jet projection area, therefore statistically the diameter of the jet varies in a certain range. It provides chances for the abrasive particles along the brim of the jet to impact and cut the hole wall at low-impact angles. Along with the AWJ drilling progress, the abrasive particles scattered beyond its border would also cause an enlargement of the hole. With a higher pressure, the hole diameter is also slightly larger. It is interesting to note that under the same condition, the hole diameters for the two materials are very close.

For a better precision and quality of hole drilling using AWJ, the precision and quality of the abrasive waterjet stream is crucial. The jet should be provided as uniform and stable as 
possible. A longer abrasive mixing tube is helpful to produce a more coherent waterjet stream. The workpiece should be mounted and aligned in a stable way to reduce the influence of impact induced vibration and misalignment. The stand-off distance between the nozzle and the part should be maintained as close as possible. Using a relatively high pressure can generate a large power jet, which achieves faster hole drilling and with less wall waviness.

It can be concluded that the workpiece mechanical properties have a direct influence on AWJ drilling characteristics such as drilling depth, material removal rate and penetration rate. Nevertheless, it is observed that the material properties have limited significance on the diameter of the holes.

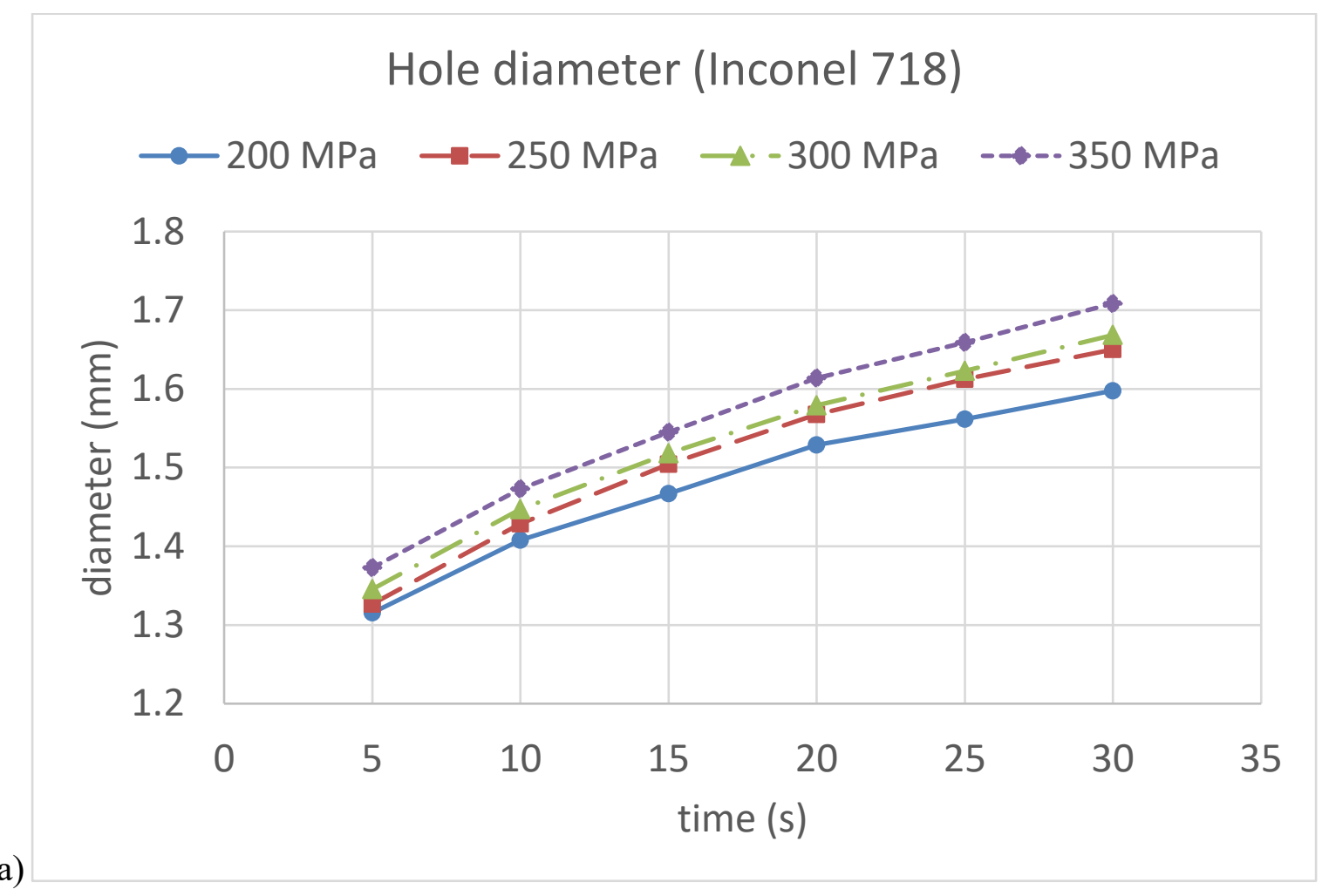




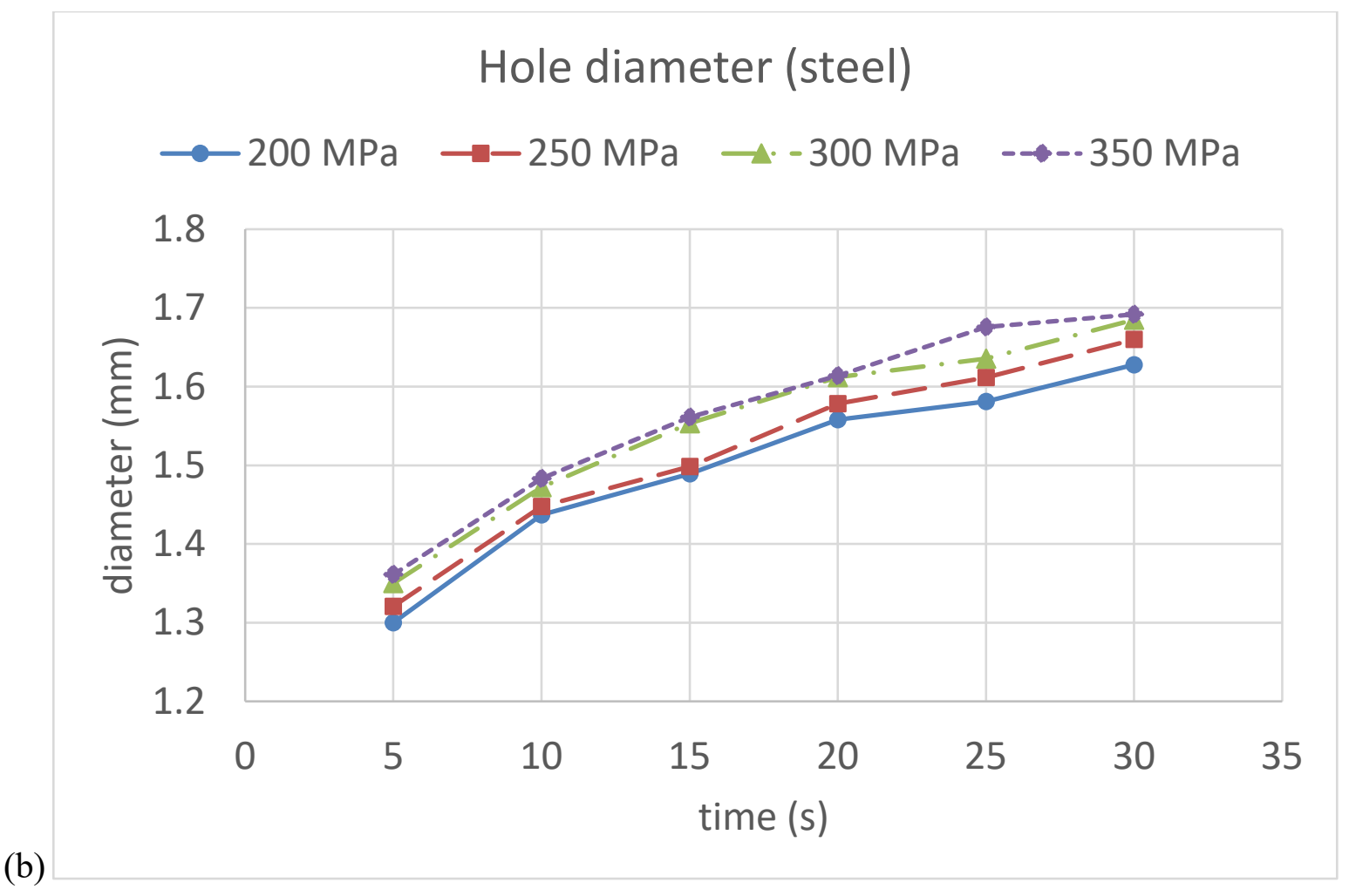

Fig. 5. Variation of the hole diameter vs drilling time for (a) Inconel 718, and (b) 1040 steel

In the AWJ drilling testing, it is not practical to detach the workpiece for weight measurement between every experiment run, as constant calibration has to be performed. Therefore, the volume of the material removed can only be approximated by using the hole depth and an estimated hole cross section to calculate. As the hole is tiny and both materials are non-transparent, measuring the taper angles of the walls of the drilled blind holes is difficult. Considering the backflow effects in piercing the blind hole, the taper can be either positive or negative. For simplicity, the hole can be approximated to be nearly a cylinder and the surface diameter of the hole be used to calculate the volume.

\subsection{Acoustic emission and process monitoring}


Acoustic emission (AE) signals were recorded to monitor the AWJ hole drilling process. AE is commonly defined as a transient elastic wave during the rapid release of energy from localized sources within a material. The generation of AE in metals is closely related to the dislocation movement accompanying plastic deformation and with the initiation and extension of cracks in a structure under stress, and also twinning, martensitic phase transformation, friction and fracture. During the AWJ cutting operation, when the abrasive waterjet exerts impacts on the target material and applies a stress on it, a strain is induced in the material $[22,23]$. Depending on the magnitude of the stress and the properties of the material, the object may experience elastic deformation, in which the original dimensions will be resiled, or plastic deformation which means it is permanently deformed after the stress is removed, or crack generation and propagation. When the material is loaded at or near its yield stress, plastic deformation occurs, which involves the movement of dislocations with atomic planes slip past each other. Such movements release energy in the form of acoustic emission which travels through the object and can be picked up by an AE sensor [27, 41].

The AE-RMS signal is believed to be able to provide more straightforward and useful information related to the process condition. According to Sutowski et al. [42], the results of AE-RMS measurements can be deemed to be representative for an analysis of the kinetic energy of the working medium. A typical waveform of the acquired AE-RMS signal acquired in the experiments is shown in Fig. 6 (a). It exhibits a general variation trend associated with an AWJ hole drilling process. It can be seen that the drilling started at the time $A$ and stopped at time $B$. The highest value of the AE-RMS signal occurred at the beginning of the AWJ piercing when the drilling just started. Due to the sudden impact from the abrasive jet at the first instance of impingement drilling, a high spike in the AE-RMS curve is observed. This phenomenon agrees with what Sutowski et al. [24] observed that AE reached its highest acoustic intensity when the jet entered (punched through) the material. After that, the AE- 
RMS signal decreased as time progressed. The general trend looks similar to the hole penetration rate curve which reflected the material removal rate. It is clear that the AWJ drilling process involves impacting the target material with an abrasive-laden waterjet with high speed to penetrate the material by erosion. The initial spike in the AE-RMS curve is a result of the collimated jet impinging the workpiece at its first instance.

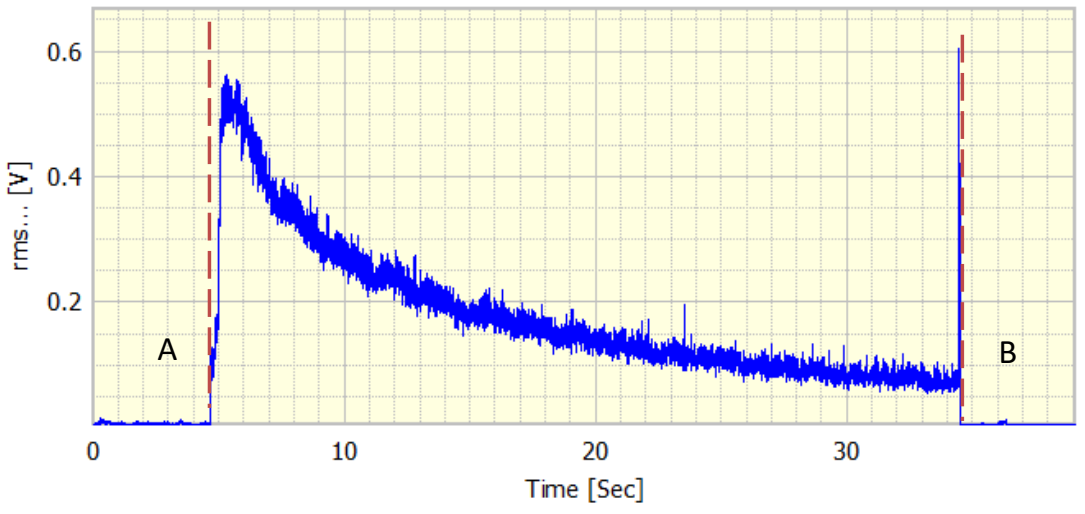

(a)

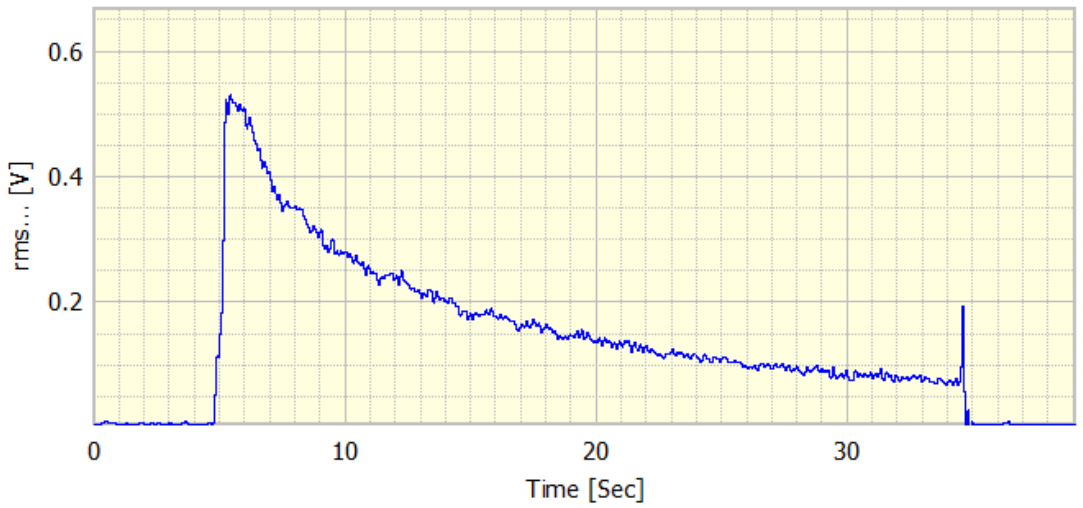

(b)

Fig. 6. Typical waveform of the AE signals in AWJ drilling. (a) AE-RMS signal, and (b) low-pass filtered AE-RMS signal

The energy available for erosion is only a portion of the total jet energy, and the portion decreases when the hole becomes deeper and deeper. It can be attributed to the energy dissipation within the blind hole and the loss of erosion efficiency due to jet backflow. Since the process starts with creating a blind hole, there exists forceful upward ejection of the jet out of the blind cavity. Along with the drilling process, the velocity of the abrasive particles 
in the jet decreases due to the existence of viscous drag force and the backflow. The kinetic energy of the jet decreases when drilling time progresses as part of the energy is lost in the fluid damping phenomena. Thus the material removal capacity of the abrasive jet decreases when the hole depth increases. The AE-RMS signal reflects such a trend in the AWJ drilling process. As the depth of penetration increases, the damping effect increases and results in a decrease in AE signal. The spike at the end of the drilling process can be possibly attributed to the instantaneous material removal at the hole walls by the back flow of the abrasive jet at the final stage. When the AWJ is switched off, fluid stops entering the blind hole and what is left is the backflow of the jet. The abrasives in the backflow no longer experiences pressure acting against it from the entering jet, and is thus able to remove more material due to higher energy levels.

Considering the fact that the AE-RMS signal is relatively noisy with remarkable ripples, a low-pass filter (LPF) was further applied to the AE-RMS signal to make the AE-RMS smoother, which facilitated the analysis and monitoring. The LPF used was a Butterworth type with a number of pass of 4 , and a cut-off frequency of $6 \mathrm{~Hz}$. The filtered AE-RMS signal is shown in Fig. 6 (b).

Fig. 7 illustrates the AE-RMS curves for AWJ drilling of Inconel 718 under the pressure of $250 \mathrm{MPa}$, over different drilling time settings $(5 \mathrm{~s}, 10 \mathrm{~s}, 15 \mathrm{~s}, \ldots, 30 \mathrm{~s})$. It is seen that the overall trend of these curves agrees very well, though the individual AE-RMS amplitude may take different values from run to run at the same time instance. For the purpose to correlate with the results of the penetration rate as presented in Fig. 5, mean values corresponding to the time instances of $2.5 \mathrm{~s}, 7.5 \mathrm{~s}, \ldots, 27.5 \mathrm{~s}$, were taken. The results of the mean AE-RMS for the two materials under the pressures of $250 \mathrm{MPa}$ and $350 \mathrm{MPa}$ are shown in Fig. 8 (a), and the corresponding penetration rates are shown in Fig. 8 (b). For each work material, under the same cutting condition, the higher the pressure, the higher the AE-RMS value. Under the 
same pressure, the AE-RMS value for Inconel 718 is much greater than that for the 1040 steel, almost more than double, while the corresponding penetration rate for Inconel 718 is significantly smaller than that for the 1040 steel. The AE-RMS reflects the decreasing trend of the penetration rate along with the drilling time, the influence of the pressure, on the other hand, it is also substantially affected by the material properties.

A comparison of the penetration rate and AE-RMS values in AWJ hole drilling of Inconel 718 under different conditions is listed in Table 7. A correlation analysis was conducted to calculate the correlation coefficient between two measurement variables, namely, the penetration rate and AE-RMS values, under each pressure level. The resultant coefficients are listed at the bottom row for each pair of penetration rate and AE-RMS values. The correlation coefficient provides a measure of the extent to which two measurement variables "vary together." The value of any correlation coefficient is in the range of -1 and +1 inclusive. A value close to 1 indicated a strong positive correlation, which means that the two measurement variables tend to move together. In this table, the resultant correlation coefficient values are between 0.86 to 0.94 under each pressure level, it demonstrated a strong correlation between the penetration rate and AE-RMS values in AWJ hole drilling, thus supported the application of AE-RMS signal as a monitoring indicator for the AWJ drilling process. It should be admitted that more effort is still required to work out a reliable model to mathematically express the relationship considering a full set of condition parameters. 


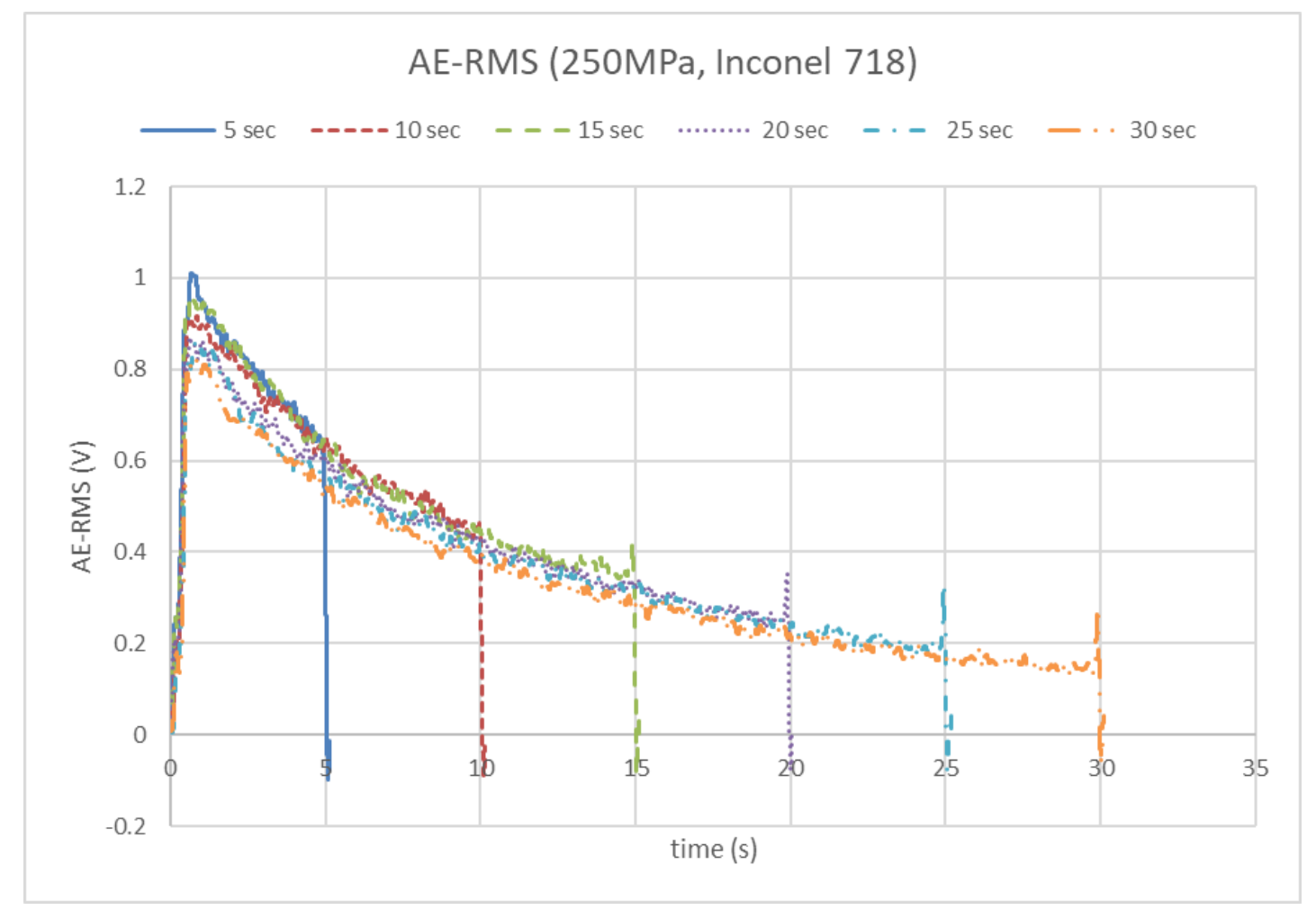

Fig. 7. Overlap of AE-RMS curves under different drilling time. Material: Inconel 718; Pressure: 250 MPa

AE-RMS was found to be a promising tool to monitor process anomalies in the AWJ drilling. In addition to such a general trend, some discrepancy of the recorded AE-RMS under similar condition is also observed. A slight variation in pressure is easily picked up by the AE sensor. An example is shown in Fig. 9, which is for AWJ drilling of the 1040 steel under $300 \mathrm{MPa}$. Among the 6 AE-RMS curves corresponding to the 6 different time length settings, the curve for the drilling period of 20 seconds shows an obvious abnormal trend comparing to others. This curve suggests an anomaly in this cutting run, that something might be abnormal or wrong. Theoretically six sets of drilling experiments with the same pressure settings were performed without any adjustments in between experiments. However, the intensifier pump was found to be inefficient in perpetuating the preset pressure. This finding agrees with [43] that the waterjet process is not entirely deterministic due to the inherent instabilities of the process; the water pressure is varying due to the nature of the intensifier pump. Other factors 
that might have affected the process include the abrasive feed that is normally fluctuating and is dependent on the moisture of the air. Pneumatic pressure in the abrasive hopper can also vary giving different amount of abrasives. It should be pointed out that the location of the AE sensor away from the workpiece plays a scaling factor effect on the AE-RMS values. A testrun and calibration for the AE signals is thus necessary.

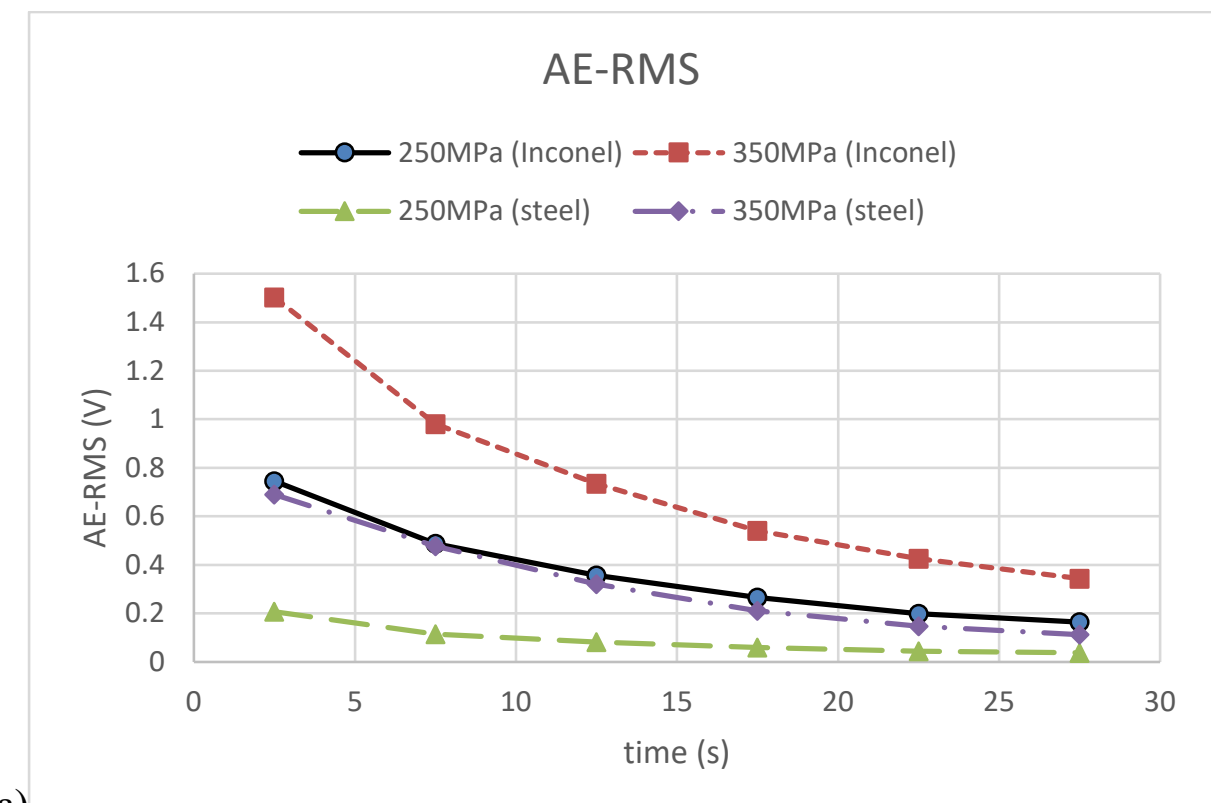

(a)

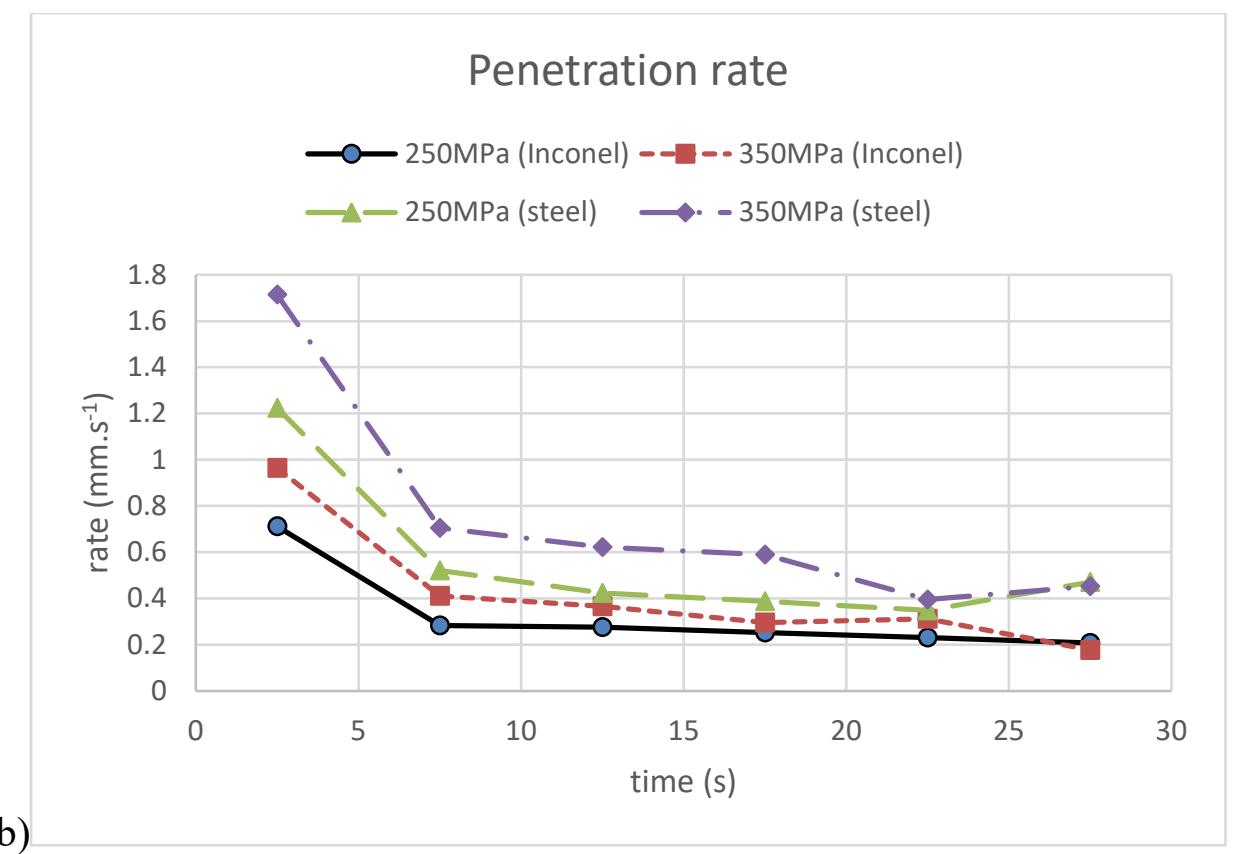

Fig. 8. Mean AE-RMS for Inconel and steel under the pressures of $250 \mathrm{MPa}$ and $350 \mathrm{MPa}$; and (b) the corresponding penetration rate 


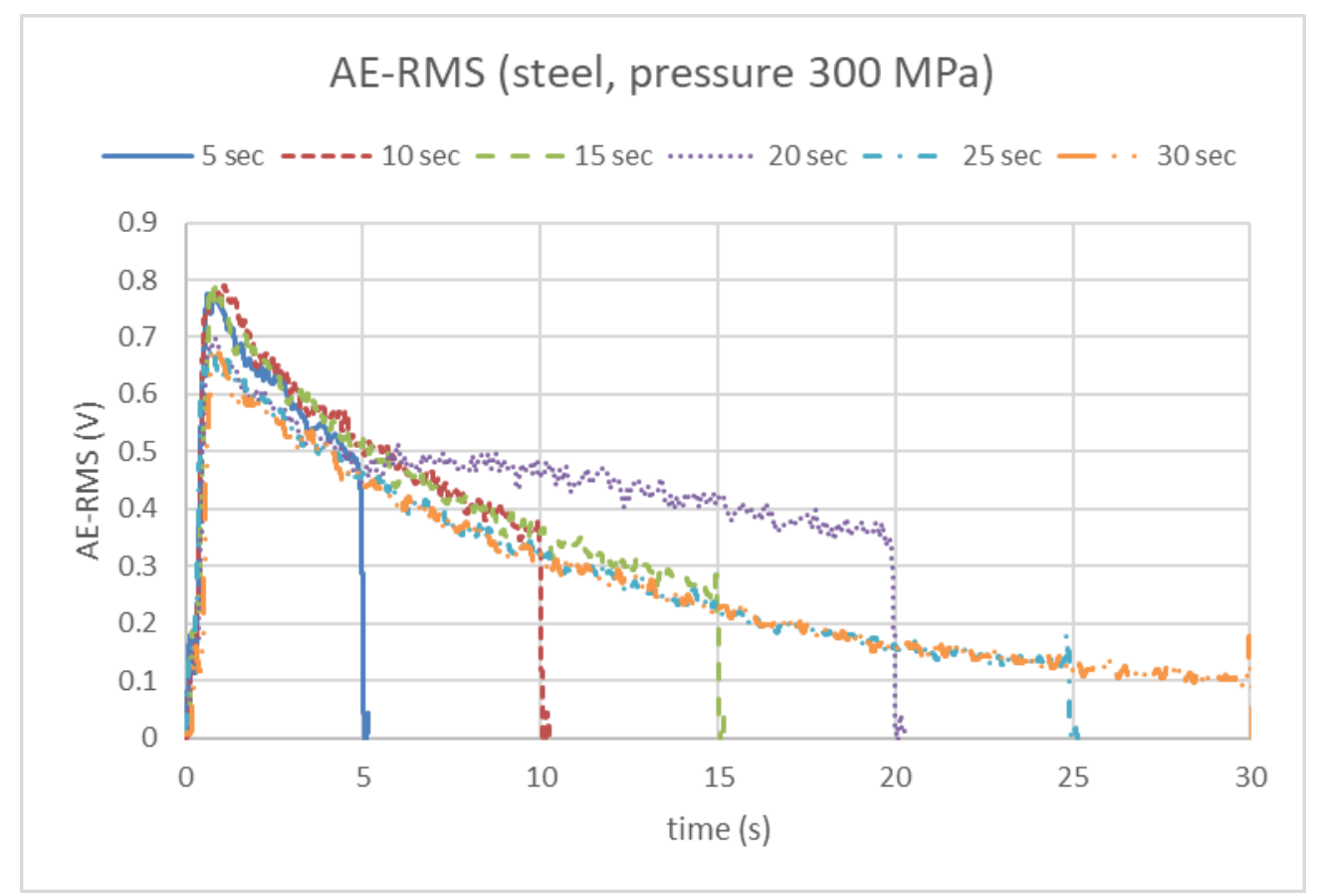

Fig. 9. Example of the AE-RMS curves with an obvious abnormal trend shown. Process condition: AWJ drilling of the 1040 steel under $300 \mathrm{MPa}$

Table 7. A comparison of the penetration rate and AE-RMS values, and their correlation, in AWJ hole drilling of Inconel 718

\begin{tabular}{c|cc|cc|cc|cc}
\hline \multirow{2}{*}{ time (s) } & \multicolumn{2}{|c|}{$200 \mathrm{MPa}$} & \multicolumn{2}{c|}{$250 \mathrm{MPa}$} & \multicolumn{2}{c|}{$300 \mathrm{MPa}$} & \multicolumn{2}{c}{$350 \mathrm{MPa}$} \\
\cline { 2 - 9 } & $\begin{array}{c}\text { Penetration } \\
\text { Rate } \\
(\mathrm{mm} / \mathrm{s})\end{array}$ & $\begin{array}{c}\mathrm{AE}- \\
\mathrm{RMS}\end{array}$ & $\begin{array}{c}\text { Penetration } \\
\text { Rate } \\
(\mathrm{mm} / \mathrm{s})\end{array}$ & $\begin{array}{c}\mathrm{AE}- \\
\mathrm{RMS}\end{array}$ & $\begin{array}{c}\text { Penetration } \\
\text { Rate } \\
(\mathrm{mm} / \mathrm{s})\end{array}$ & $\begin{array}{c}\text { AE- } \\
\text { RMS }\end{array}$ & $\begin{array}{c}\text { Penetration } \\
\text { Rate } \\
(\mathrm{mm} / \mathrm{s})\end{array}$ & $\begin{array}{c}\text { AE- } \\
\text { RMS }\end{array}$ \\
\hline 2.5 & 0.584 & 0.299 & 0.712 & 0.552 & 0.818 & 0.597 & 0.964 & 1.149 \\
7.5 & 0.276 & 0.217 & 0.284 & 0.400 & 0.368 & 0.424 & 0.412 & 0.803 \\
12.5 & 0.216 & 0.159 & 0.276 & 0.305 & 0.306 & 0.334 & 0.366 & 0.591 \\
17.5 & 0.206 & 0.122 & 0.252 & 0.218 & 0.320 & 0.242 & 0.296 & 0.487 \\
22.5 & 0.176 & 0.093 & 0.232 & 0.168 & 0.348 & 0.169 & 0.312 & 0.408 \\
27.5 & 0.184 & 0.062 & 0.208 & 0.122 & 0.196 & 0.133 & 0.178 & 0.311 \\
\hline Correlation & \multicolumn{2}{|c|}{0.899} \\
\hline
\end{tabular}

\section{Conclusions}

In this paper, an experimental study on abrasive waterjet drilling of both Inconel 718 and AISI 1040 steel is presented, with an aim to understand the AWJ hole drilling performance 
and to investigate the process monitoring by using acoustic emission. The following conclusions can be made:

- The hole depth increases along with the drilling time at a slowing down rate. The higher the water pressure, the deeper the hole depth. It was found that the power regression curve exhibits the best correlation coefficient for the experimental data.

- The penetration rate decreases along with the drilling time and the increasing hole depth. This can be attributed to the loss of the kinetic energy of the jet with an increasing depth, due to the existence of viscous drag force and a backflow of the impacting jet in a blind hole produced.

- Due to the wall erosion effects resulting from the backflow in hole drilling, the hole diameter generated is considerably wider than the nozzle size and the jet stream.

- There exists a direct influence of the material property (strength) on the AWJ drilling efficiency. The penetration rate for the steel is much higher than that for the Inconel under same cutting condition. However, the material property has been confirmed to have limited significance with regards to the diameter of the hole produced.

- AE-RMS signal has been demonstrated to be of value in monitoring the AWJ drilling process. It reflects the decreasing trend of the penetration rate along with the drilling time, and the influence of the pressure. A correlation analysis showed a strong correlation between the penetration rate and AE-RMS values in AWJ hole drilling, thus supported the application of AE-RMS signal as a monitoring indicator. On the other hand, the AE-RMS value is also substantially affected by the material properties. Under same condition, the AE-RMS for Inconel 718 is much greater than that for the steel, which is in contrast to the lower penetration rate for Inconel 718.

- A discrepancy of the AE-RMS under similar condition may suggest an anomaly in this cutting run. Since the location of the AE sensor can affect the AE-RMS values, a 
test-run and calibration for the AE signals is recommended for a specific AWJ machining process monitoring.

\section{Acknowledgement}

The author would like to thank Mr Clement Wong for his contribution to this study. The author would like to express his acknowledgments to the Advanced Manufacturing Laboratory, UNSW, for the support of the experimental work.

\section{Declarations of interest: none'}

This research did not receive any specific grant from funding agencies in the public, commercial, or not-for-profit sectors.

\section{References}

1. Li HZ, Chen XQ (2013) Tool Condition Monitoring in Machining. In: Zhang S, Zhao D (eds) Aerospace Materials Handbook. CRC Press, pp 77-108

2. Chen XQ, Li HZ (2009) Development of a tool wear observer model for online tool condition monitoring and control in machining nickel-based alloys. Int J Adv Manuf Technol 45:786-800. https://doi.org/10.1007/s00170-009-2003-1

3. Li HZ, Zeng H, Chen XQ (2006) An experimental study of tool wear and cutting force variation in the end milling of Inconel 718 with coated carbide inserts. J Mater Process Technol 180:296-304. https://doi.org/10.1016/j.jmatprotec.2006.07.009

4. Li H, Wang J (2012) A cutting forces model for milling Inconel 718 alloy based on a material constitutive law. Proc Inst Mech Eng Part C J Mech Eng Sci 227:1761-1775. https://doi.org/10.1177/0954406212469167 
5. Wanner B, Archenti A, C.M. N (2018) Hybrid Machining: An Industrial Case-Study Comparing Inconel718 Reaming and Drilling with Abrasive Waterjet Technology. In:

Ni J, Majstorovic V, Djurdjanovic D (eds) Proceedings of 3rd International

Conference on the Industry 4.0 Model for Advanced Manufacturing. AMP 2018.

Lecture Notes in Mechanical Engineering. Springer, pp 109-114

6. Hashish M (2017) CUTTING AND SHAPING OF THICK MATERIALS WITH AWJ. Wjta-Imc 1-11

7. Rabani A, Madariaga J, Bouvier C, Axinte D (2016) An approach for using iterative learning for controlling the jet penetration depth in abrasive waterjet milling. J Manuf Process 22:99-107. https://doi.org/10.1016/j.jmapro.2016.01.014

8. Li H, Wang J (2015) An experimental study of abrasive waterjet machining of Ti-6Al4V. Int J Adv Manuf Technol 81:361-369. https://doi.org/10.1007/s00170-015-7245-5

9. Wang J (1999) Machinability study of polymer matrix composites using abrasive waterjet cutting technology. J Mater Process Technol 94:30-35. https://doi.org/10.1016/S0924-0136(98)00443-9

10. Zhong ZW, Han ZZ (2003) Performance comparison of four waterjet nozzles. Mater Manuf Process 18:965-978. https://doi.org/10.1081/AMP-120025082

11. Supriya SB, Srinivas S (2018) Machinability Studies on Stainless steel by abrasive water jet -Review. Mater Today Proc 5:2871-2876.

https://doi.org/10.1016/j.matpr.2018.01.079

12. Wang J (2007) Predictive depth of jet penetration models for abrasive waterjet cutting of alumina ceramics. Int J Mech Sci 49:306-316. https://doi.org/10.1016/j.ijmecsci.2006.09.005 
13. Gudimetla P, Wang J, Wong W (2002) Kerf formation analysis in the abrasive waterjet cutting of industrial ceramics. J Mater Process Technol 128:123-129

14. Boud F, Carpenter C, Folkes J, Shipway PH (2010) Abrasive waterjet cutting of a titanium alloy: The influence of abrasive morphology and mechanical properties on workpiece grit embedment and cut quality. J Mater Process Technol 210:2197-2205. https://doi.org/10.1016/j.jmatprotec.2010.08.006

15. Mieszala M, Torrubia PL, Axinte DA, et al (2017) Erosion mechanisms during abrasive waterjet machining : Model microstructures and single particle experiments. J Mater Process Tech 247:92-102. https://doi.org/10.1016/j.jmatprotec.2017.04.003

16. Beer N, Özkaya E, Biermann D (2014) New production technologies in aerospace industry - 5th machining innovations conference (MIC 2014) drilling of inconel 718 with geometry-modified twist drills. Procedia CIRP 24:49-55. https://doi.org/10.1016/j.procir.2014.07.124

17. Kovacevic R, Fang M (1994) Modeling of the influence of the abrasive waterjet cutting parameters on the depth of cut based on fuzzy rules. Int J Mach Tools Manuf 34:55-72. https://doi.org/https://doi.org/10.1016/0890-6955(94)90040-X

18. Axinte DA, Srinivasu DS, Billingham J, Cooper M (2010) Geometrical modelling of abrasive waterjet footprints: A study for 908 jet impact angle. CIRP Ann - Manuf Technol 59:341-346. https://doi.org/10.1016/j.cirp.2010.03.112

19. Zeng J, Kim TJ (1993) Parameter prediction and cost analysis in abrasive waterjet cutting operations. In: Proceedings of the 7th American Water Jet Conference. Seattle, Washington, pp 175-189

20. Ramulu M, Posinasetti P, Hashish M (2005) Analysis of the Abrasive Waterjet 
Drilling Process. WJTA Am Waterjet Conf 5-2

21. Lissek F, Kaufeld M, Tegas J, Hloch S (2016) Online-monitoring for abrasive waterjet cutting of CFRP via acoustic emission: Evaluation of machining parameters and work piece quality due to burst analysis. Procedia Eng 149:67-76.

https://doi.org/10.1016/j.proeng.2016.06.640

22. Rabani A, Marinescu I, Axinte D (2012) Acoustic emission energy transfer rate: A method for monitoring abrasive waterjet milling. Int J Mach Tools Manuf 61:80-89. https://doi.org/10.1016/j.ijmachtools.2012.05.012

23. CHOI GS, CHOI GH (1997) Process analysis and monitoring in abrasive water jet machining of alumina ceramics. Int J Mach Tools Manuf 37:295-307

24. Sutowski P, Sutowska M, Kapłonek W (2018) The use of high-frequency acoustic emission analysis for in-process assessment of the surface quality of aluminium alloy 5251 in abrasive waterjet machining. Proc Inst Mech Eng Part B J Eng Manuf 232:2547-2565. https://doi.org/https://doi.org/10.1177/0954405417703428

25. Mokhtar N, Gebremariam MA, Zohari H, Azhari A (2018) Analysis of acoustic emission during abrasive waterjet machining of sheet metals. IOP Conf Ser Mater Sci Eng 342:. https://doi.org/10.1088/1757-899X/342/1/012107

26. Pahuja R, Ramulu M (2018) Abrasive waterjet process monitoring through acoustic and vibration signals. 24th Int Conf Water Jet 75-87

27. Hassan AI, Chen C, Kovacevic R (2004) On-line monitoring of depth of cut in AWJ cutting. Int J Mach Tools Manuf 44:595-605. https://doi.org/10.1016/j.ijmachtools.2003.12.002 
28. AISI 1040 Carbon Steel (UNS G10400).

https://www.azom.com/article.aspx?ArticleID=6525. Accessed 6 Jun 2019

29. Li W (2013) A study of abrasive waterjet turning process and impact erosion by high velocity micro-particles. The University of New South Wales

30. Pawar PJ, Vidhate US, Khalkar MY (2018) Improving the quality characteristics of abrasive water jet machining of marble material using multi-objective artificial bee colony algorithm. J Comput Des Eng 5:319-328.

https://doi.org/10.1016/J.JCDE.2017.12.002

31. Wright I An Engineer's Guide to Waterjet Cutting. https://www.engineering.com/AdvancedManufacturing/ArticleID/12716/AnEngineers-Guide-to-Waterjet-Cutting.aspx. Accessed 6 Jun 2019

32. Hocheng H, Chang KR (1994) Material removal analysis in abrasive waterjet cutting of ceramic plates. J Mater Process Technol 40:287-304. https://doi.org/10.1016/09240136(94)90456-1

33. Nguyen T, Wang J (2019) A review on the erosion mechanisms in abrasive waterjet micromachining of brittle materials. Int J Extrem Manuf 1:012006. https://doi.org/10.1088/2631-7990/ab1028

34. Orbanic H, Jurisevic B, Kramar D, et al (2006) Miniaturization of injection abrasive water jet machining process. Proc Inst Mech Eng Part C J Mech Eng Sci 220:16971705. https://doi.org/10.1243/09544062JMES217

35. Zhong ZW, Han ZZ (2002) Turning of glass with abrasive waterjet. Mater Manuf Process 17:339-349. https://doi.org/10.1081/AMP-120005380 
36. Natarajan Y, Murugesan PK, Mohan M, et al (2020) Abrasive Water Jet Machining process : A state of art of review. J Manuf Process 49:271-322.

https://doi.org/10.1016/j.jmapro.2019.11.030

37. Khan AA, Haque MM (2007) Performance of different abrasive materials during abrasive water jet machining of glass. J Mater Process Technol 191:404-407. https://doi.org/10.1016/j.jmatprotec.2007.03.071

38. Fowler G, Pashby IR, Shipway PH (2009) The effect of particle hardness and shape when abrasive water jet milling titanium alloy Ti6A14V. Wear 266:613-620. https://doi.org/10.1016/j.wear.2008.06.013

39. Axinte DA, Srinivasu DS, Kong MC, Butler-Smith PW (2009) Abrasive waterjet cutting of polycrystalline diamond: A preliminary investigation. Int J Mach Tools Manuf 49:797-803. https://doi.org/10.1016/j.ijmachtools.2009.04.003

40. Li HZ, Wang J, Fan JM (2009) Analysis and modelling of particle velocities in microabrasive air jet. Int J Mach Tools Manuf 49:850-858. https://doi.org/10.1016/j.ijmachtools.2009.05.012

41. Lissek F, Kaufeld M, Tegas J, Hloch S (2016) Online-monitoring for abrasive waterjet cutting of CFRP via acoustic emission : Evaluation of machining parameters and work piece quality due to burst analysis. Procedia Eng 149:67-76. https://doi.org/10.1016/j.proeng.2016.06.640

42. Sutowski P, Plichta J, Kałduński P (2019) Determining kinetic energy distribution of the working medium in a centrifugal disc finishing process - part 2: experimental analysis with the use of acoustic emission signal. Int J Adv Manuf Technol 104:687704. https://doi.org/10.1007/s00170-019-03937-2 
43. Fredin J, Jönsson A (2011) Experimentation on Piercing with Abrasive Waterjet.

World Acad Sci Eng Technol 5:1663-1669 


\section{List of Figures}

Fig. 1. (a) The robot manipulator and the cutting head nozzle for the AWJ system; (b) Locations of workpiece and the AE sensor in the preliminary testing; (c) Acquired AE signal with sensor saturation; (d) Final locations of workpiece and the AE sensor

Fig. 2. (a) Sample photo of the garnet particles under microscope; (b) Nozzle movement procedures in the AWJ drilling experiments; (c) a resultant blind hole; (d) Vertex 420 for hole diameter measurement

Fig. 3. Hole depth vs. the drilling time for (a) Inconel 718, and (b) 1040 steel, under different conditions

Fig. 4. Segmented estimation of the penetration rate for AWJ drilling of (a) Inconel 718, and (b) 1040 steel

Fig. 5. Variation of the hole diameter vs drilling time for (a) Inconel 718, and (b) 1040 steel Fig. 6. Typical waveform of the AE signals in AWJ drilling. (a) AE-RMS signal, and (b) lowpass filtered AE-RMS signal

Fig. 7. Overlap of AE-RMS curves under different drilling time. Material: Inconel 718; Pressure: $250 \mathrm{MPa}$

Fig. 8. Mean AE-RMS for Inconel and steel under the pressures of $250 \mathrm{MPa}$ and $350 \mathrm{MPa}$; and (b) the corresponding penetration rate

Fig. 9. Example of the AE-RMS curves with an obvious abnormal trend shown. Process condition: AWJ drilling of the 1040 steel under $300 \mathrm{MPa}$ 


\section{List of Tables}

Table 1. Inconel 718 chemical composition (\%)

Table 2. AISI 1040 chemical composition (\%)

Table 3. Fixed Process Parameters in AWJ drilling

Table 4. Ratio of the hole depth for the two materials (steel / Inconel) under each condition

Table 5. Ratio of material properties (Inconel/ steel)

Table 6. Measured diameter and circularity under different cutting conditions for Inconel 718

Table 7. A comparison of the penetration rate and AE-RMS values, and their correlation, in AWJ hole drilling of Inconel 718 